\title{
The vascular flora of Porto Ferreira State Park: an ecotonal area in São Paulo State, southeastern Brazil
}

\author{
Gabriel Pavan Sabino $^{1 *}{ }^{\circledR}$, Vitor de Andrade Kamimura ${ }^{1,2} \mathbb{D}$, Renan Borgiani ${ }^{1}$, Rafael Konopczyk ${ }^{1}$, Ernesto Pedro \\ Dickfeldt ${ }^{3}$, José Eduardo de Arruda Bertoni ${ }^{4}$, Sonia Aparecida de Souza Evangelista ${ }^{3}$ \& Gabriel Mendes Marcusso ${ }^{1}$ (i) \\ ${ }^{1}$ Universidade Estadual Paulista "Júlio de Mesquita Filho”, Instituto de Biociências, Departamento de \\ Botânica, Av. 24, 13506-000, Rio Claro, SP, Brasil. \\ ${ }^{2}$ Universidade Federal de Minas Gerais Departamento de Botânica, Instituto de Ciências Biológicas, Av. \\ Antônio Carlos, 31270-901, Belo Horizonte, MG, Brasil. \\ ${ }^{3}$ Instituto Florestal, Rua do Horto, 02377-000, São Paulo, SP, Brasil. \\ ${ }^{4}$ Instituto Agronomico de Campinas, Divisão de horticultura, Seção de Floricultura e Plantas Ornamentais, \\ Rua Barão de Itapura, 13020-902, Campinas, SP, Brasil. \\ *Corresponding author: gpsabino@gmail.com
}

SABINO, G.P., KAMIMURA, V.A., BORGIANI, R., KONOPCZYK, R., DICKFELDT, E.P., BERTONI, J.E.A., EVANGELISTA, S.A.S., MARCUSSO, G.M. The vascular flora of Porto Ferreira State Park: an ecotonal area in São Paulo State, southeastern Brazil. Biota Neotropica. 21(4): e20211229. https://doi.org/10.1590/1676-0611-BN-2021-1229

\begin{abstract}
The Porto Ferreira State Park (PFSP) is located in the State of São Paulo southeastern Brazil, in an intriguing transitional area between the Atlantic Forest and Cerrado - both hotspots of biodiversity - represented mainly by the cerradão (CER), and the seasonal semideciduous forest (SSF), with its alluvial variation vegetation type (riparian forest - RP). Ecotonal areas play an important role in providing ecological and phytogeographic knowledge regarding the flora and vegetation of this region. Despite various studies on the PFSP, knowledge of this region remains fragmented. In this study, we aim to conduct an updated checklist of the PFSP vascular flora, including a compilation of all the studies conducted in this protected area, plus field work carried out by the authors from 2014 to 2017. In addition, given its ecotonal characteristics, we completed a floristic similarity analysis between the PFSP and other floristic surveys that examined the same vegetation types present in this study, to gain a better understanding of their phytogeographic relationships. Overall, 684 species, belonging to 387 genera and 107 families, were recorded. The SSF presented the richest vegetation type (478 species), followed by the CER (418) and the RP (231). The most diverse families were Fabaceae (64 species), Myrtaceae (41), Orchidaceae (39), Rubiaceae (37), Asteraceae (35), Bignoniaceae (26) and Malvaceae (20). Moreover, eight threatened species, at regional and national levels, were found. To date, 412 species have been added to the floristic list produced for the PFSP. The life forms with the highest number of species were trees (286 species), herbs (176) plus shrubs and subshrubs (123). Our research findings indicate floristic patterns with higher levels of similarity among species in geographical proximity, including those in ecotonal areas encompassing different vegetation types. These results rank the PFSP among some of the most species-rich conservation units with seasonal climates, and therefore is of great importance for plant conservation in the southeast of Brazil.
\end{abstract}

Keywords: Atlantic Forest; Cerrado; floristic; protected area; seasonal semideciduous forest.

\section{Flora vascular do Parque Estadual de Porto Ferreira: uma área ecotonal no estado de São Paulo, sudeste do Brasil}

Resumo: O Parque Estadual de Porto Ferreira (PEPF) está localizado no estado de São Paulo, sudeste do Brasil, em uma intrigante área de contato entre a Mata Atlântica e o Cerrado - ambos hotspots de biodiversidade -, representada principalmente pelo cerradão (CER), a floresta estacional semidecidual (FES) e pela variação dessa última, com influência aluvial (FESA). As áreas ecotonais desempenham um papel importante no conhecimento ecológico e fitogeográfico da flora e da vegetação. Mesmo com muitos estudos realizados no PEPF, as informações encontram-se dispersas. Dessa forma, produzimos um checklist atualizado da flora vascular, resultante da compilação de vouchers, estudos realizados no PEPF e de esforços de coleta realizados pelos autores entre os anos de 2014 e 2017. Além disso, para entender suas relações fitogeográficas, realizamos uma análise de similaridade florística entre o PEPF e outros levantamentos realizadas nas mesmas fitofisionomias da área estudada. Registramos 684 espécies pertencentes a 387 gêneros e 107 famílias. A FES foi a fitofisionomia mais rica (478 espécies), seguida pelo CER (418) e a FESA (231). 
As famílias mais ricas foram Fabaceae (64 espécies), Myrtaceae (41), Orchidaceae (39), Rubiaceae (37), Asteraceae (35), Bignoniaceae (26) e Malvaceae (20). Oito espécies ameaçadas em âmbito regional e nacional foram encontradas. Desde a última lista florística produzida para o PEPF, 412 espécies foram adicionadas. As formas de vida com maiores números de espécies foram: árvores (286 espécies), ervas (176) e arbustos mais subarbustos (123). A análise de similaridade corroborou o padrão de que áreas mais próximas geograficamente são mais similares entre si, e esse padrão foi encontrado, também, para áreas ecotonais envolvendo diferentes fitofisionomias. Os resultados encontrados colocam o PEPF entre as unidades de conservação com maior número de espécies vegetais sob o domínio de clima sazonal, possuindo, portanto, grande importância para a conservação de espécies de plantas no Sudeste do Brasil.

Palavras-chave: Mata Atlântica; Cerrado; florística; unidade de conservação; floresta estacional semidecidual.

\section{Introduction}

Brazil is home to one of the richest plant diversities in the world, with more than 34,000 species of vascular plants (BFG, 2021). This great diversity is mainly due to Brazil's vast geographical area, its heterogeneous topography, as well as its climate, and biogeographical features (Fiaschi \& Pirani, 2009). Traditionally, it has been divided into major landscapes and macroecological ecoregions (Ab'Sáber, 1970, 2003). Among the biogeographical units recognized in Brazil, the Cerrado and the Atlantic Forest are classified as biodiversity hotspots for global conservation, hosting an overwhelming number of endemic species threatened by anthropic actions (Myers et al., 2000; Mittermeier et al., 2004).

The biogeographic province, known as the Cerrado, mainly spans over central Brazil, with its southern limits reaching the northern part of the State of Paraná, the northern region of Argentina, and eastern Bolivia (Morrone, 2017; Velazco et al., 2018). It comprises a set of vegetation types, ranging from grasslands with herbaceous plants dominating the landscape (campo limpo), to savannas (campo sujo and cerrado sensu stricto) and forests (e.g. cerradão and gallery forests) (Coutinho, 2006; Batalha, 2011). The Cerrado is the second largest phytogeographical unit of Brazil, spanning 2 million $\mathrm{km}^{2}$ or $23.9 \%$ of the national territory (IBGE, 2004). The main threats to the Cerrado vegetation are the expansion of intensive agriculture activity, and raising livestock (Ratter et al., 2006; Klink \& Moreira, 2002). Nevertheless, the Cerrado is considered one of the world's richest savanna vegetation (Klink \& Machado, 2005; Simon et al. 2009), containing more than 12,000 species of vascular plants (BFG, 2015; Flora do Brasil, 2020). In the State of São Paulo the Cerrado occurs mainly in the central region, but extends to eastern portions of the State as well, with enclaves in the Atlantic Forest matrix (Baitello et al., 2013).

The Atlantic Forest covers nearly 1.1 million $\mathrm{km}^{2}$ (IBGE, 2004), distributed over a broad latitudinal, ranging from the State of Piauí to Rio Grande do Sul (SOS Mata Atlântica, 2016), and reaching inland to northern Argentina and eastern Paraguay (Spichiger et al., 2006; Fiaschi \& Pirani, 2009). Brazil's Atlantic Forest hosts an overwhelming species richness of vascular plants (BFG 2015; Flora do Brasil, 2020), being one of the world's top five species-rich areas (Barthlott et al., 2005). Traditionally, the Atlantic Forest has been considered a single biogeographical unit with subdivisions (e.g. Morellato \& Haddad, 2000). However, more than distinct vegetation types, singular biogeographical histories of the biota have been suggested for different parts of the Atlantic Forest (Vieira et al., 2015; Morrone 2014, 2017; Eisenlohr \& Oliveira-Filho, 2015) justifying its division in three provinces (Morrone 2014, 2017).
Among these biogeographical provinces, the inland and seasonal vegetation type is represented by the Paraná Forest province (Morrone, 2017; Zanotti et al., 2020), and it is the one most threatened by the reduction of the original area (Ribeiro et al., 2009). The floristic richness of the seasonal forest is considerably high (Souza et al., 2019a) and holds regional and typical species (Eisenlohr \& Oliveira-Filho, 2015; Vieira et al., 2015; Morrone 2014, 2017).

In the State of São Paulo, the Cerrado and Atlantic Forest mix in a complex and transitional area, in scattered patches of each or at ecotones (Durigan et al., 2012). The Cerrado forest formation is very common there, with a great richness of tree species, mainly influenced by contact with Atlantic Forest taxa (Françoso et al., 2016). On the other hand, the innermost vegetation type of the Atlantic Forest is characterized by a well-marked dry season, and a characteristic composition, despite sharing several species with the rainy coastal forest and the cerradão (Eisenlohr \& Oliveira-Filho, 2015). Ecotones represent a transition between ecological communities, with a mixture of the floristic and faunistic characteristics of two different and relatively homogenous ecological community types (Allen \& Starr, 1982). Studies indicate that richness and abundance tend to peak at ecotonal areas (Kark \& van Rensburg, 2006) and contribute to São Paulo's high level of plant richness, having one of Brazil's greatest numbers of species (BFG, 2015; Flora do Brasil, 2020).

Studies on the biodiversity of plant communities are considered fundamental, and one of the first steps toward the establishment of models for the preservation and conservation of ecosystems (Morellato \& Leitão-Filho, 1992; Ferreira Júnior et al., 2008). Given these characteristics, the PFSP, floristically, has drawn great attention. From 1984 to present, 12 studies regarding its flora have been carried out, as well as aleatory collections deposited in herbaria. These studies include climbing plants (Vargas et al., 2018), ferns (Colli et al., 2003), non-arboreal plants (Oliveira, 2012; Osaco, 2012), trees (Bertoni, 1984; Bertoni \& Martins, 1987; Bertoni et al., 2001; Sabino, 2013; Konopczyk, 2014) and vascular epiphytes (Marcusso et al., 2016). However, this data is fragmented, and remains to be systematically organized. The Management Plan (São Paulo, 2003) represents the last overall floristic list presented for this protected area.

Thus, we compiled the checklist of the vascular flora of the PFSP, based on fieldwork, studies conducted in the area, and herbarium collections, aiming to extend and systematize the scattered floristic information on the region. This data can support restoration and conservation projects in the central region of the State of São Paulo, given that several private lands of the region present a deficit in native vegetation and must be restored in accordance with the Native Vegetation Protection Law (Tavares et al., 2019). 
Our work also provides a foundation on which to update the Management Plan of this important vegetation-protected area. In addition, we explored and discussed the floristic identity concerning the ecotonal condition of the PFSP, comparing our data with other floristic surveys carried out on the same vegetation types.

\section{Material and Methods}

The Porto Ferreira State Park (PFSP), known locally as "Mata do Procópio", is located in the municipality of Porto Ferreira, of the State of São Paulo, in the southeastern region of Brazil (Figure 1). The PFSP is one of more than 50 integral protected areas in São Paulo (Instituto Florestal/Fundação Florestal, 2008, Colli-Silva et al., 2016), founded in 1962, initially categorized as a State Reserve, and later (1987) becoming a State Park (São Paulo, 2003). Under the central geographical coordinates $21^{\circ} 51^{\prime} \mathrm{S}$ and $47^{\circ} 25^{\prime} \mathrm{W}$, the park is bordered by the Mogi Guaçu river to the south, and by the SP-215 highway to the north, occupying an area of 611.55 hectares (São Paulo, 2003). The PFSP is part of the region of the Peripheral Depression of São Paulo (Depression of Mogi Guaçu) belonging to the Paraná Basin (Rossi et al., 2005).
According to Köeppen-Geiger classification, the climate of the region is classified as Cwa, dry winter mesothermal (Alvares et al., 2013). The annual mean temperature is $22^{\circ} \mathrm{C}$, and the annual mean precipitation is $1470 \mathrm{~mm}$ (INMET 2019).

The vegetation of the Mogi Guaçu basin is mainly composed of Atlantic Forest, with seasonal semideciduous forest (SSF) being the most common vegetation type (Bertoni 1984; Veloso \& Góes Filho, 1982). However, PFSP is composed of different vegetation types, presenting the forested savanna in the north (CER, with altitudes between 605-575 m), and to the south, SSF (with altitudes between 575-535 m) (Rossi et al., 2005). Parallel to the Mogi Guaçu river, riparian forest is found (RP, with an average altitude of $530 \mathrm{~m}$ ) (Bertoni \& Martins, 1987; Konopczyk, 2014) (Figure 2). These vegetation types can be distinguished mainly by their structures, of which the CER presents a small average height and high density, while the SSF presents a greater average height and lower density (Vargas et al. 2018), and finally, the RP having the lowest density and diversity (Konopczyk, 2014). In addition, there are ecotonal areas among them (Sabino, 2013), as well as riverine variations (Konopczyk, 2014).
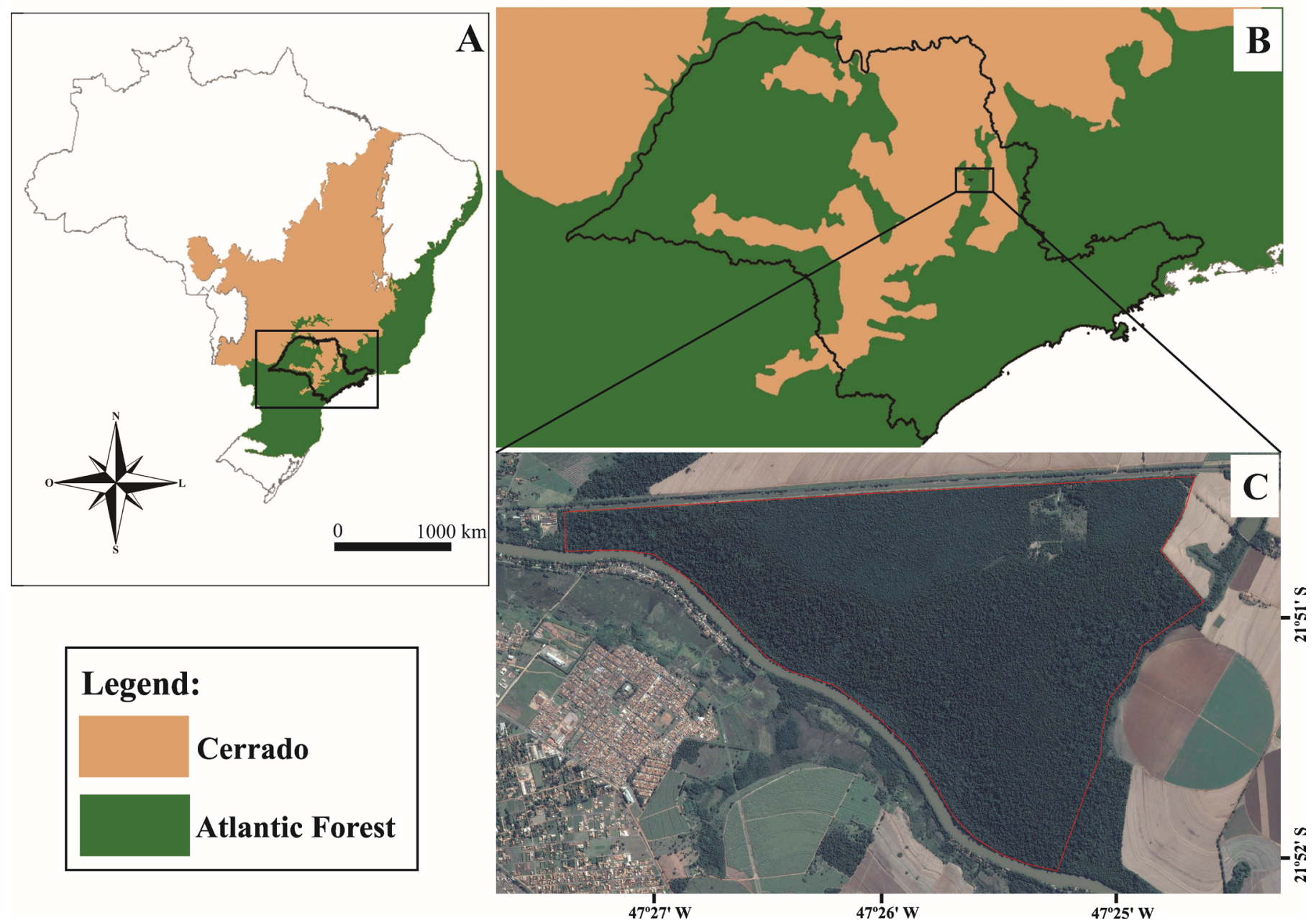

Figure 1. Location of Porto Ferreira State Park (PFSP), Porto Ferreira, São Paulo State. A. Atlantic Forest and the Cerrado in Brazil and the location of São Paulo State; B. Location of PFSP; C. PFSP satellite view, adapted from Google Earth. 

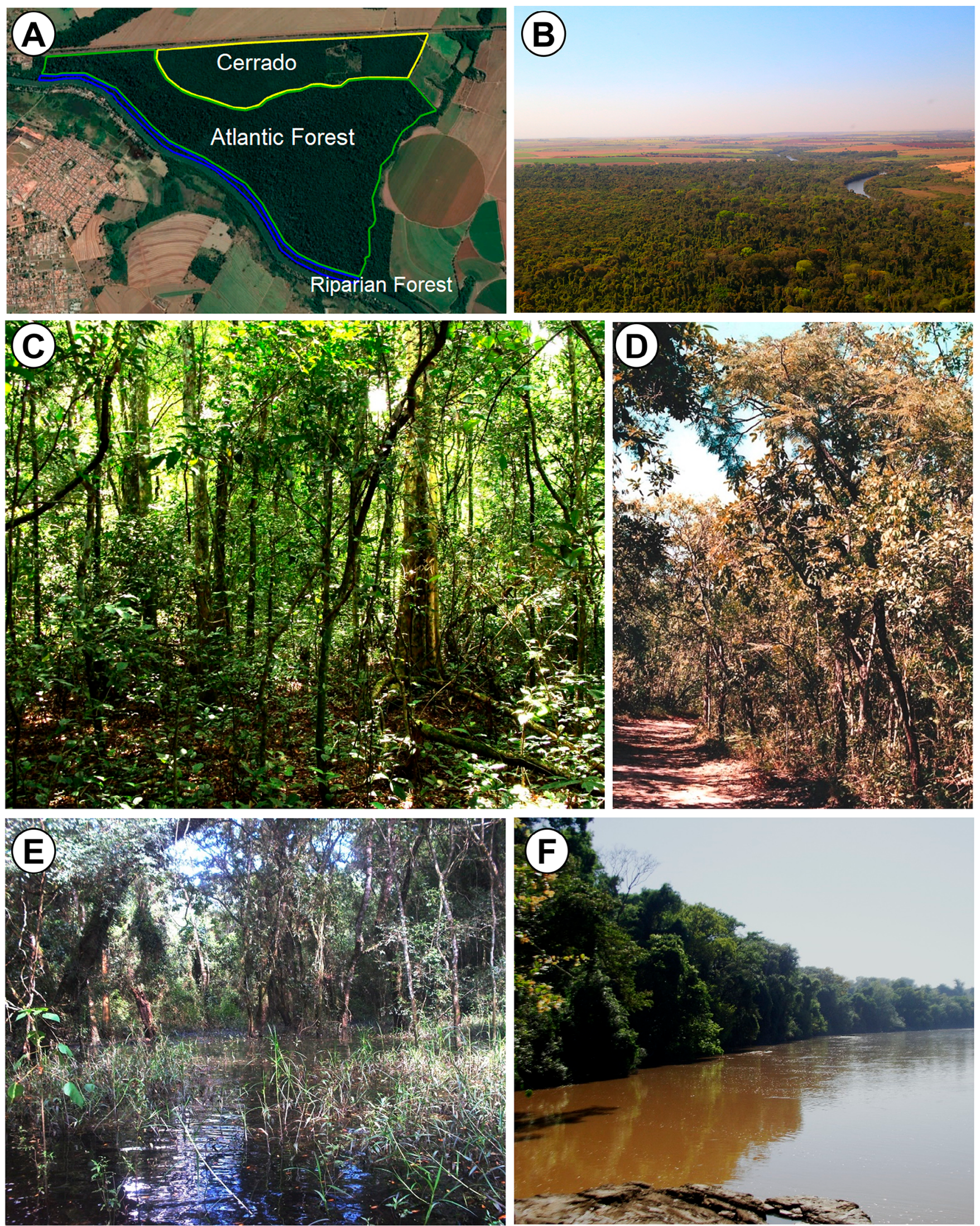

Figure 2. Vegetation types of Porto Ferreira State Park (PFSP), Porto Ferreira, São Paulo State. A: map indicating the vegetation types; B: aerial view of PFSP; C: seasonal semideciduous forest; D: cerradão; E: riparian forest; F: Mogi Guaçu river (A. Adapted from Google Earth; B. PFSP photo collection; C./F. Gabriel Mendes Marcusso; D. PFSP photo collection; E. Rafael Konopczyk). 
The PFSP represents one of the few protected areas in São Paulo which comprises the contact between the Cerrado and Altantic Forest, becoming an important biotic pool of both, in a single and relatively small area. It also holds one of the largest and tallest populations of Cariniana legalis (Mart.) Kuntze and Cariniana estrellensis (Raddi) Kuntze (Lecythidaceae), with several individuals reaching up to $50 \mathrm{~m}$. These trees are iconic elements of the PFSP.

The PFSP vascular flora checklist was created from the following: specimens collected by the authors from 2014 to 2017, using the walking research method (Filgueiras et al., 1994); the systematization of all other lists found in surveys carried out in PFSP(Bertoni, 1984; Bertoni \& Martins, 1987; Bertoni et al., 2001; São Paulo, 2003; Colli et al., 2003; Oliveira, 2012; Osaco, 2012; Dickfeldt et al., 2013; Sabino, 2013; Konopczyk, 2014; Marcusso et al., 2016; Vargas et al., 2018); and the compilation of vouchers present in the Specieslink database (CRIA, 2019). We considered only native taxa identified at the taxonomic level of species and excluded those with dubious identification, at the genera-level and exotic species (cited in a separate table - Appendix 1). For angiosperms, the taxa classification followed the APG IV system (2016), and for ferns or lycophytes, the PPG I system (2016). The nomenclature and synonymizations were updated according to the Flora do Brasil (2020). When the data from Flora do Brasil (2020) were outdated or not available, we used the Tropicos (2019) database.
The life forms of the species were obtained through field observations, assessing the herbarium specimen labels information and, when this was not possible, we consulted the Flora do Brasil (2020) data. Shrubs and subshrubs were unified in a single category. To assess the number of exclusive and common species among the vegetation types in the study area, we built Venn diagrams for all the species together, and for each life form. The threatened species were classified according to The Red Book of Brazilian Flora (Martinelli \& Moraes, 2013) and the list of threatened flora of São Paulo (SMA-SP, 2016).

The similarity in species composition was estimated by the Jaccard Index (Mueller-Dombois \& Ellenberg, 1974), as well as this study and 14 others carried out in Cerrado sensu lato, SSF and RP vegetation types. Only vascular or phanerogamic floristic surveys were compared, which included studies performed in the South and Southeast regions of Brazil. Only native taxa identified at the taxonomic level of species were considered. Studies that sampled more than one vegetation type were subdivided into independent lists (e.g. Durigan et al., 1999), resulting in 22 lists (Table 1). We used a binary matrix with presence (1) and absence $(0)$ to conduct an agglomerative hierarchical clustering analysis (Legendre \& Legendre, 2012), by employing UPGMA (average linkage), and Jaccard dissimilarity in the vegan package (Oksanen et al. 2013) in R (R Core Team 2020).

Table 1. Studies of flora compared with this study.

\begin{tabular}{|c|c|c|c|c|}
\hline $\begin{array}{l}\text { CODE FOR THE } \\
\text { STUDIES }\end{array}$ & STUDY SITE & VEGETATION TYPE & $\mathbf{N S a} / \mathbf{N}$ & REFERENCE \\
\hline $\mathrm{A}$ & Porto Ferreira - SP & Ssf & $478 / 478$ & Present study \\
\hline $\mathrm{C}$ & Porto Ferreira - SP & $\mathrm{Rp}$ & $231 / 231$ & Present study \\
\hline $\mathrm{D}$ & Piracicaba - SP & Ssf & $269 / 289$ & Corrêa et al. 2018 \\
\hline $\mathrm{F}$ & Campinas - SP & Ssf & $187 / 201$ & Guaratini et al. 2008 \\
\hline G & Descoberto - MG & Ssf & $648 / 708$ & Forzza et al. 2014 \\
\hline $\mathrm{H}$ & São João Nepomuceno - MG & Ssf & $174 / 204$ & Pifano et al. 2013 \\
\hline I & Caratinga - MG & Ssf & $826 / 1048$ & Lombardi \& Gonçalves 2000 \\
\hline M & Avaré - SP & $\mathrm{Ec}$ & $33 / 33$ & Cielo-Filho et al. 2015 \\
\hline $\mathrm{N}$ & Santa Rita do Passa Quatro - SP & Cer & $145 / 148$ & Batalha \& Mantovani 2001 \\
\hline $\mathrm{O}$ & Santa Rita do Passa Quatro - SP & Css & $336 / 339$ & Batalha \& Mantovani 2001 \\
\hline $\mathrm{P}$ & Assis - SP & Cer & $214 / 242$ & Durigan et al. 1999 \\
\hline Q & Assis - SP & $\mathrm{Rp}$ & $120 / 134$ & Durigan et al. 1999 \\
\hline $\mathrm{R}$ & Assis - SP & Css & $222 / 298$ & Durigan et al. 1999 \\
\hline $\mathrm{S}$ & Pirassununga - SP & Csl & $339 / 358$ & Batalha et al. 1997 \\
\hline $\mathrm{T}$ & Pratânia - SP & Css & $113 / 120$ & Carvalho et al. 2010 \\
\hline $\mathrm{U}$ & Bauru - SP & Csl & $360 / 371$ & Cavassan \& Weiser 2015 \\
\hline
\end{tabular}

Ssf: seasonal semideciduous forest; Rp: riparian forest; Cer: cerradão; Csl: cerrado sensu lato; Css: cerrado sensu stricto; Cc: campo cerrado; Ec: ecotone Ssf-Cer; NSa: number of taxons used in similarity analysis; N: total number of taxons. 


\section{Results}

A total of 684 species distributed in 107 families and 387 genera were recorded (640 angiosperms and 44 ferns or lycophytes) (Table 2). The richest families were Fabaceae, with 64 species, followed by Myrtaceae (41), Orchidaceae (39), Rubiaceae (37), Asteraceae (35), Bignoniaceae (26), Malvaceae (20), Euphorbiaceae (19), Malpighiaceae and Sapindaceae (17 each) (Figure 3). These families account for $46 \%$ of the surveyed species, and 35 families $(32.7 \%)$ had one single species. The richest genera were Eugenia (19 species), Miconia (14), Myrcia (11), Solanum (nine), Peperomia and Serjania (eight each). A separate list shows 152 excluded species and the reason for the exclusion (Appendix 1). The richest vegetation type was SSF, with 478 species, followed by the CER (418) and the RP (231).
From the registered species, 581 were listed in previous surveys, and 527 of them we found in herbaria vouchers. Added to the last floristic list prepared for the management plan, were 412 species, 70 of which were drawn from aleatory collections (not cited in previous surveys), and 33 from collections carried out by the authors of this study. Eight species recorded here are on the threatened list at the national level (Martinelli \& Moraes, 2013) two of which - Anemopaegma arvense (Vell.) Stellfeld ex de Souza and Cariniana legalis (Mart.) Kuntze - are endangered, and six species [Euterpe edulis Mart., Zeyheria tuberculosa (Vell.) Bureau ex Verl., Croton leptobotryus Müll.Arg., Cedrela fissilis Vell., Cattleya walkeriana Gardner and Isabelia virginalis Barb.Rodr.] are listed as vulnerable. The list of threatened São Paulo State flora (SMA 2016) follows the same species and threat category as on the national list, except for Cariniana legalis (Mart.) Kuntze, classified as vulnerable.

Table 2. List of vascular flora species from Porto Ferreira State Park, municipality of Porto Ferreira, São Paulo State, southeastern Brazil.

\begin{tabular}{|c|c|c|c|c|}
\hline Family/Species & Life form & Voucher & Source & Veg. type \\
\hline \multicolumn{5}{|l|}{ ACANTHACEAE } \\
\hline Justicia lythroides (Nees) V.A.W.Graham & Herb & $\mathrm{HRCB}, 52297$ & IV, XIII & cer, ssf \\
\hline Ruellia brevifolia (Pohl) C.Ezcurra & Shrub & HRCB, 52389; MBML, 43930 & IV, XIII & cer, ssf \\
\hline Ruellia jussieuoides Schltdl. \& Cham. & Shrub & HRCB, 52299 & IV, XIII & cer, ssf \\
\hline Chamissoa acuminata Mart. & Shrub & HRCB, 52323; HRCB, 68052 & IV, IX, XIII & ssf \\
\hline Pfaffia tuberosa (Spreng.) Hicken & Herb & HRCB, 54948; FUEL, 53509 & IV, XIII & $\mathrm{rp}$ \\
\hline \multicolumn{5}{|l|}{ AMARYLLIDACEAE } \\
\hline Hippeastrum puniceum (Lam.) Kuntze & Herb & HRCB, 71787 & XIII & $\mathrm{rp}$ \\
\hline Lithraea molleoides (Vell.) Engl. & Tree & SPSF, 24691; UEC, 44048 & III, XIII, X & cer \\
\hline Schinus terebinthifolia Raddi & Tree & & III, $\mathrm{X}$ & cer, ssf \\
\hline Tapirira guianensis Aubl. & Tree & SPSF, 24702; UB, 147873 & I, II, III, V, VI, VIII, X, XIII & cer, ssf, rp \\
\hline Tapirira obtusa (Benth.) J.D.Mitch. & Tree & & II, VI, X & cer, ssf, rp \\
\hline \multicolumn{5}{|l|}{ ANEMIACEAE } \\
\hline Anemia phyllitidis (L.) Sw. & Herb & BHCB, 141939 & IV, XI, XIII & cer, ssf \\
\hline Anemia tomentosa var. anthriscifolia (Schrad.) Mickel & Herb & BHCB, 153399 & IV & cer, ssf \\
\hline Anemia villosa Humb. \& Bonpl. ex Willd. & Herb & & XI & cer, ssf \\
\hline \multicolumn{5}{|l|}{ ANNONACEAE } \\
\hline Guatteria australis A.St.-Hil. & Tree & SPSF, 25925 & III, X, XIII & cer, ssf, rp \\
\hline Xylopia aromatica (Lam.) Mart. & Tree & MBM, 287171; SPSF, 27379 & III, VI, X, XIII & cer \\
\hline Xylopia brasiliensis Spreng. & Tree & & III, V, VI, X & cer, ssf, rp \\
\hline Xylopia sericea A.St.-Hil. & Tree & & VI & cer \\
\hline
\end{tabular}




\begin{tabular}{|c|c|c|c|c|}
\hline Aspidosperma cylindrocarpon Müll.Arg. & Tree & & I, II, VII, X & ssf, rp \\
\hline Aspidosperma polyneuron Müll.Arg. & Tree & & II, X & ssf, rp \\
\hline Aspidosperma ramiflorum Müll.Arg. & Tree & $\mathrm{HRCB}, 71805$ & I, X, XIII & ssf \\
\hline Aspidosperma tomentosum Mart. & Tree & & III, X & cer \\
\hline Forsteronia glabrescens Müll.Arg. & Climbing & HRCB, 52508 & IV, XIII & cer, ssf \\
\hline Forsteronia pubescens A.DC. & Climbing & HRCB, 67993 & IX & cer, ssf \\
\hline Gonolobus rostratus (Vahl) R.Br. ex Shult. & Climbing & HRCB, 52643 & IV, XIII & cer \\
\hline Mandevilla pohliana (Stadelm.) A.H.Gentry & Shrub & UEC, 37775 & XIII & cer \\
\hline Secondatia densiflora A.DC. & Climbing & HRCB, 67996 & IX & cer, ssf \\
\hline Temnadenia violacea (Vell.) Miers & Climbing & HRCB, 52464; HRCB, 71791 & IV, IX, XIII & cer, ssf \\
\hline \multicolumn{5}{|l|}{ AQUIFOLIACEAE } \\
\hline Ilex cerasifolia Reissek & Tree & SPSF, 27381; MBM, 288153 & III, X, XIII & cer \\
\hline \multicolumn{5}{|l|}{ ARACEAE } \\
\hline Philodendron propinquum Schott & Herb & HRCB, 62017 & VIII, XIII & cer, ssf, rp \\
\hline $\begin{array}{l}\text { Thaumatophyllum bipinnatifidum (Schott ex Endl.) } \\
\text { Sakur., Calazans \& Mayo }\end{array}$ & Herb & $\mathrm{HRCB}, 62030$ & VIII, XIII & ssf \\
\hline \multicolumn{5}{|l|}{ ARALIACEAE } \\
\hline Acrocomia aculeata (Jacq.) Lodd. ex Mart. & Herb & & III, VI, X & cer, rp \\
\hline Allagoptera campestris (Mart.) Kuntze & Herb & & III, X & cer \\
\hline Euterpe edulis Mart.* & Herb & & I, V, VI, X & cer, ssf, rp \\
\hline Geonoma brevispatha Barb.Rodr. & Herb & & III, X & cer, rp \\
\hline Syagrus loefgrenii Glassman & Herb & SPSF, 20831 & XIII & cer \\
\hline Syagrus oleracea (Mart.) Becc. & Herb & & $\mathrm{I}, \mathrm{X}$ & ssf, rp \\
\hline Syagrus romanzoffiana (Cham.) Glassman & Herb & & I, II, III, VI, X & cer, ssf, rp \\
\hline \multicolumn{5}{|l|}{ ARISTOLOCHIACEAE } \\
\hline Aristolochia labiata Willd. & Climbing & HRCB, 52506; HRCB, 67998 & IV, IX, XIII & cer, ssf \\
\hline \multicolumn{5}{|l|}{ ASPARAGACEAE } \\
\hline Herreria latifolia Woodson & Climbing & SPSF, 26298 & XIII & cer, ssf \\
\hline \multicolumn{5}{|l|}{ ASPLENIACEAE } \\
\hline Asplenium auriculatum Sw. & Herb & UEC, 62342 & XIII & ssf \\
\hline Asplenium claussenii Hieron. & Herb & APO, 100 & IV, XI & ssf, rp \\
\hline Asplenium formosum Willd. & Herb & UEC, 62341 & XI, XIII & ssf \\
\hline Asplenium inaequilaterale Willd. & Herb & & $\mathrm{XI}$ & ssf \\
\hline Asplenium otites Link & Herb & & XI & ssf \\
\hline
\end{tabular}




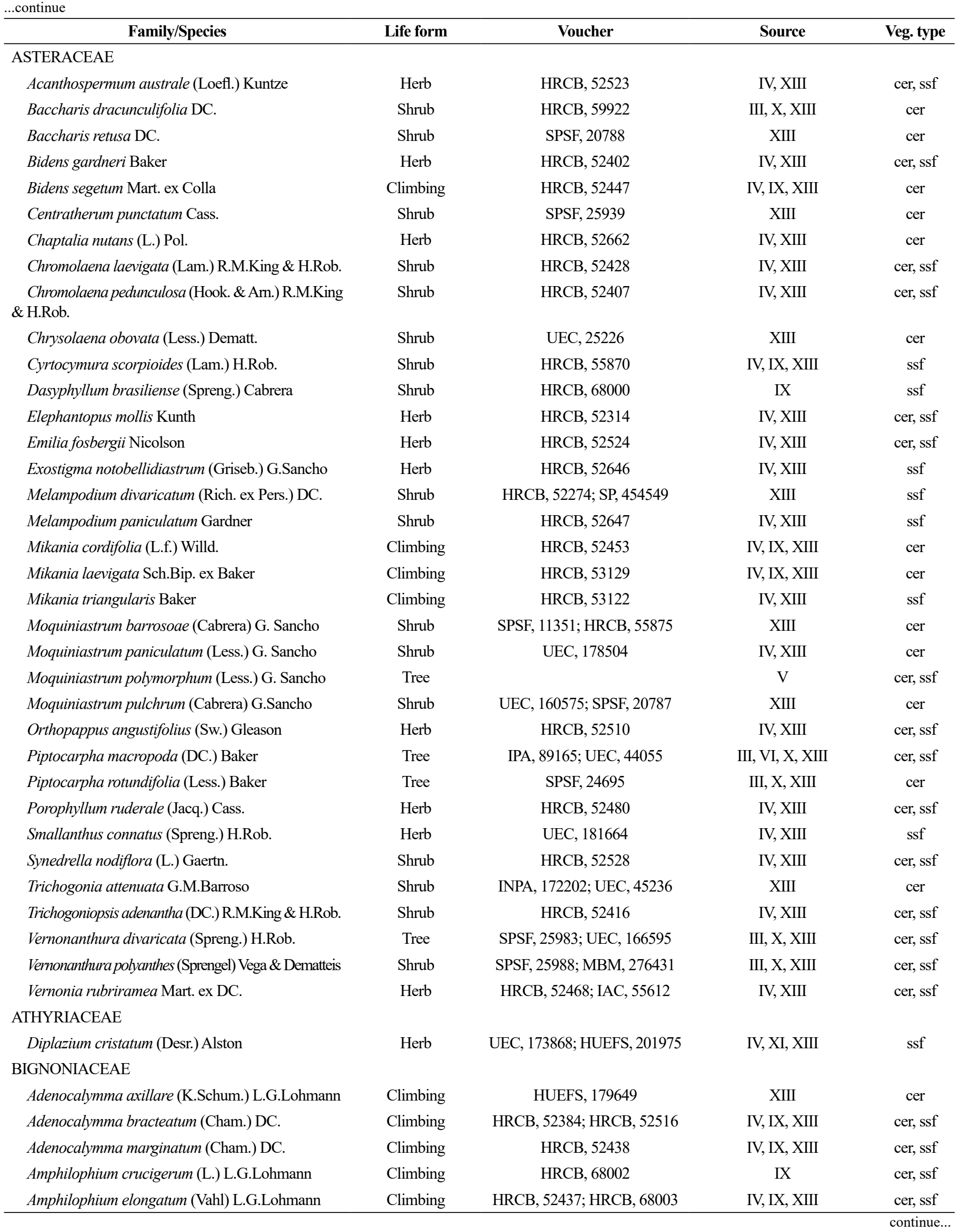




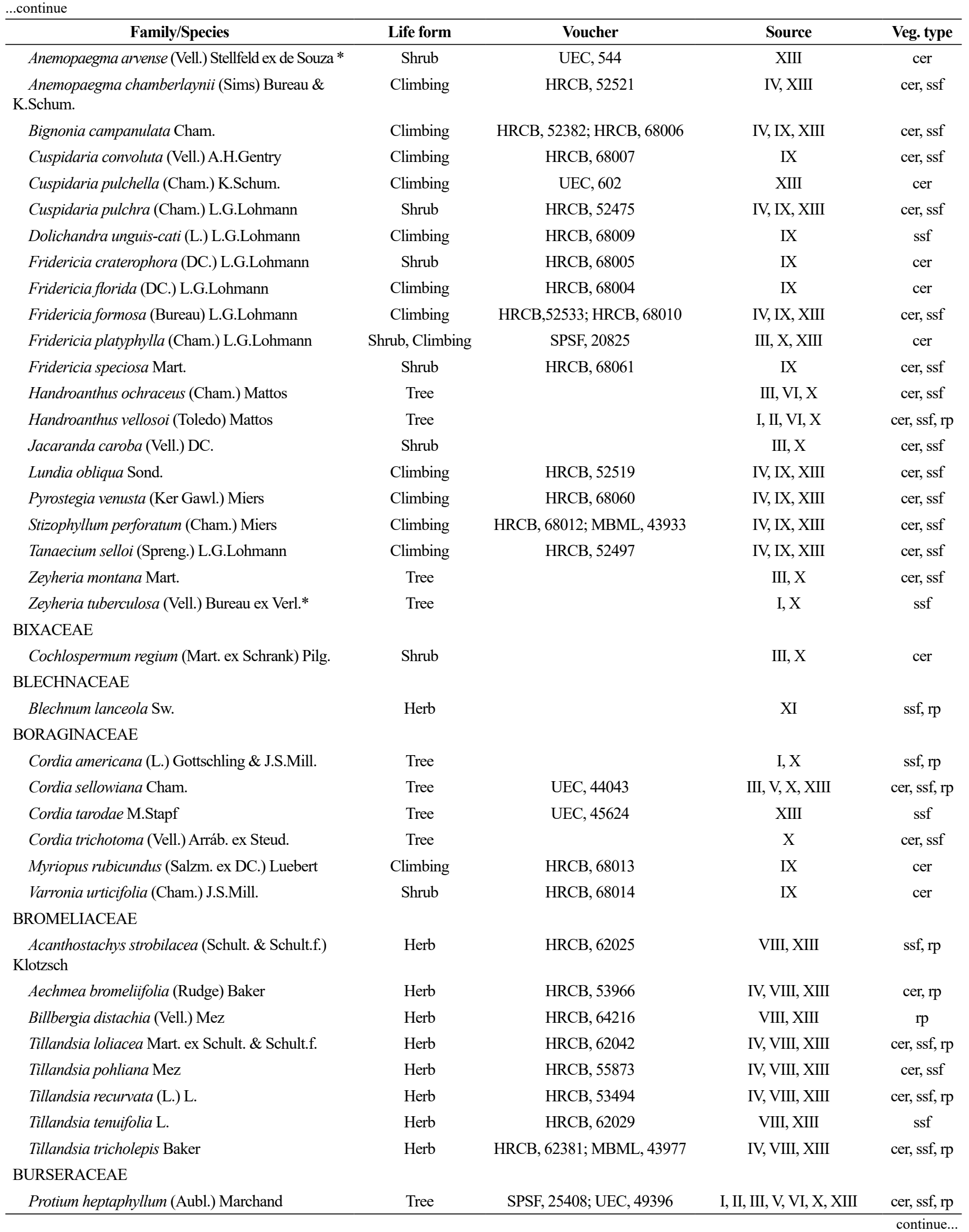




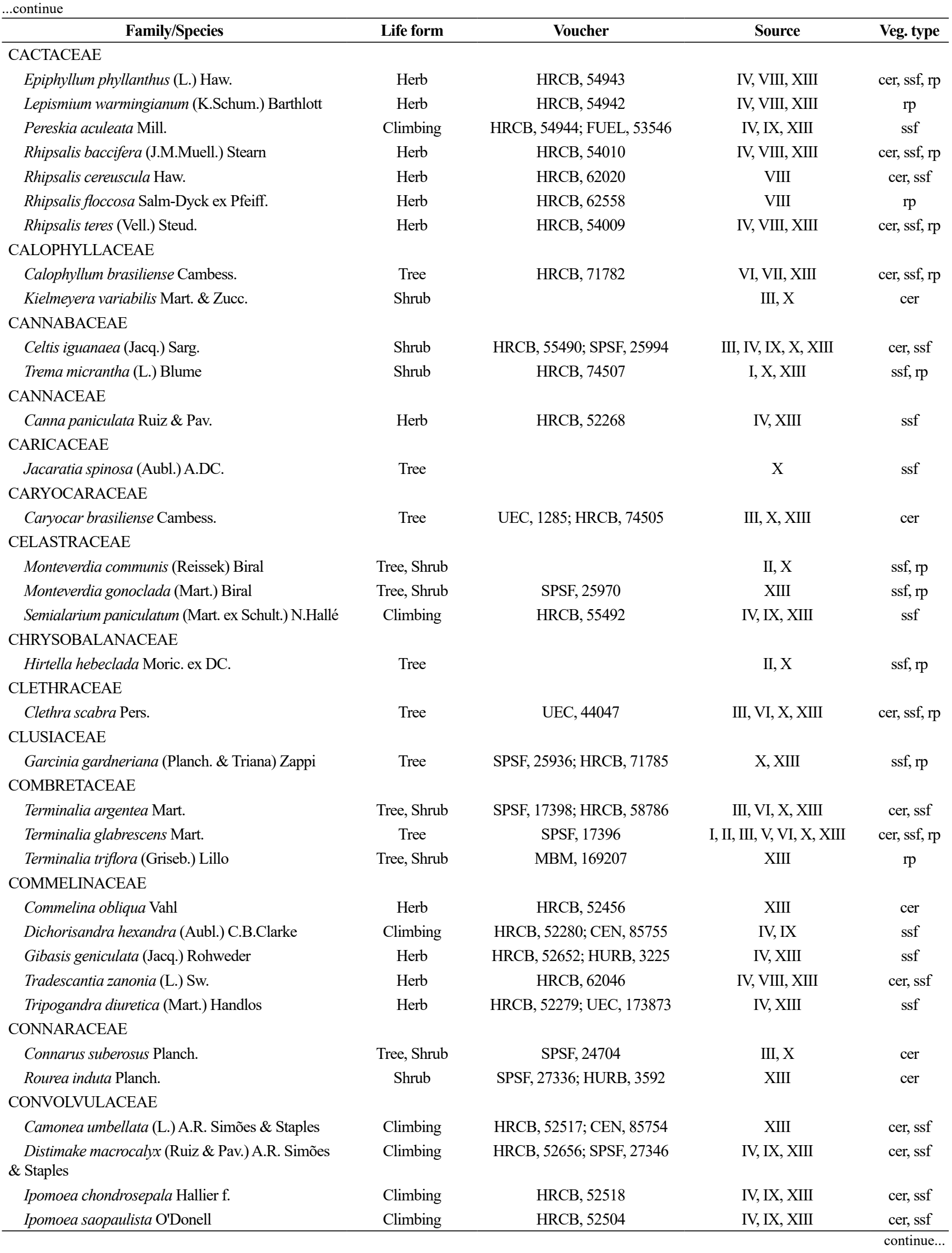


Flora of Porto Ferreira State Park

\begin{tabular}{|c|c|c|c|c|}
\hline Psiguria ternata (M.Roem.) C.Jeffrey & Climbing & HRCB, 68016 & IX & cer \\
\hline Wilbrandia hibiscoides Silva Manso & Climbing & UEC, 43383 & XIII & ssf \\
\hline \multicolumn{5}{|l|}{ CUNONIACEAE } \\
\hline Lamanonia ternata Vell. & Tree & UEC, 75747 & III, X, XIII & cer, ssf \\
\hline Cyathea delgadii Sternb. & Herb & & XI & $\mathrm{rp}$ \\
\hline \multicolumn{5}{|l|}{ CYPERACEAE } \\
\hline Cyperus laxus Lam. & Herb & HRCB, 52433 & IV, XIII & cer, ssf \\
\hline Cyperus luzulae (L.) Retz. & Herb & HRCB, 54933 & IV, XIII & $\mathrm{rp}$ \\
\hline Scleria latifolia Sw. & Herb & HRCB, 71789 & IX, XIII & ssf, rp \\
\hline \multicolumn{5}{|l|}{ DILLENIACEAE } \\
\hline Davilla elliptica A.St.-Hil. & Shrub, Climbing & HRCB, 52451 & III, IV, IX, X, XIII & cer, ssf \\
\hline Davilla rugosa Poir. & Climbing & HRCB, 52663 & IV, IX, XIII & cer, ssf \\
\hline Doliocarpus dentatus (Aubl.) Standl. & Shrub, Climbing & HRCB, 52500 & IV, IX, XIII & cer, ssf \\
\hline \multicolumn{5}{|l|}{ DIOSCOREACEAE } \\
\hline Dioscorea dodecaneura Vell. & Climbing & JAL, 7736 & IV, IX & cer, ssf \\
\hline Dioscorea olfersiana Klotzsch ex Griseb. & Climbing & HRCB, 52395 & IV, IX, XIII & cer, ssf \\
\hline \multicolumn{5}{|l|}{ DRYOPTERIDACEAE } \\
\hline Sloanea hirsuta (Schott) Planch. ex Benth. & Tree & HRCB, 4966; UEC, 25465 & XIII & ssf \\
\hline \multicolumn{5}{|l|}{ ERYTHROXYLACEAE } \\
\hline Erythroxylum buxus Peyr. & Shrub & UEC, 44051 & XIII & $\mathrm{rp}$ \\
\hline Erythroxylum cuneifolium (Mart.) O.E.Schulz & Shrub & FUEL, 39773 & III, X, XIII & cer \\
\hline Erythroxylum deciduum A.St.-Hil. & Tree, Shrub & BOTU, 20419 & V, XIII & cer, ssf \\
\hline Erythroxylum pelleterianum A.St.-Hil. & Shrub & TEPB, 25904 & III, V, X, XIII & cer, ssf \\
\hline Erythroxylum suberosum A.St.-Hil. & Tree, Shrub & SPSF, 25273 & III, X, XIII & cer \\
\hline Erythroxylum subracemosum Turcz. & Tree, Shrub & & III, X & cer \\
\hline Erythroxylum tortuosum Mart. & Tree, Shrub & & III, $\mathrm{X}$ & cer \\
\hline \multicolumn{5}{|l|}{ EUPHORBIACEAE } \\
\hline Actinostemon concepcionis (Chodat \& Hassl.) Hochr. & Shrub & HRCB, 62085; SPSF, 49093 & III, V,VII, X, XIII & cer, ssf, rp \\
\hline Actinostemon klotzschii (Didr.) Pax & Tree, Shrub & INPA, 78113 & I, II, III, X, XIII & cer, rp \\
\hline Alchornea glandulosa Poepp. \& Endl. & Tree & SPSF,25976 & III, VI, X, XIII & cer, ssf, rp \\
\hline Aparisthmium cordatum (A.Juss.) Baill. & Tree & & $\mathrm{V}$ & ssf \\
\hline Cnidoscolus urens (L.) Arthur & Shrub & SPSF, 19516; SPSF, 20820 & XIII & $\operatorname{ssf}$ \\
\hline Croton floribundus Spreng. & Tree & HRCB, 74503 & I, II, III, V, VI, X, XIII & cer, ssf, rp \\
\hline Croton leptobotryus Müll.Arg. * & Shrub & HRCB, 71804 & XIII & cer \\
\hline
\end{tabular}




\begin{tabular}{|c|c|c|c|c|}
\hline Family/Species & Life form & Voucher & Source & Veg. type \\
\hline Croton lundianus (Didr.) Müll.Arg. & Shrub & $\mathrm{HRCB}, 52414$ & IV, XIII & cer, ssf \\
\hline Croton salutaris Casar. & Tree & & $\mathrm{I}, \mathrm{X}$ & ssf, rp \\
\hline Croton urucurana Baill. & Tree & $\mathrm{HRCB}, 62052$ & I, II, VII, X, XIII & $\mathrm{rp}$ \\
\hline Dalechampia pentaphylla Lam. & Climbing & HRCB, 52431 & IV, IX, XIII & cer, ssf \\
\hline Dalechampia stipulacea Müll.Arg. & Climbing & HRCB, 52318 & IV, IX, XIII & ssf \\
\hline Dalechampia triphylla Lam. & Climbing & HRCB, 68019 & IX & ssf \\
\hline Euphorbia sciadophila Boiss. & Herb & HRCB, 52272 & IV, XIII & ssf \\
\hline Gymnanthes klotzschiana Müll.Arg. & Tree, Shrub & HRCB, 62050; SPSF, 49075 & I, II, VII, X, XIII & ssf, rp \\
\hline Mabea fistulifera Mart. & Tree, Shrub & IAC, 54530; SPSF, 20783 & III, X, XIII & cer \\
\hline Maprounea guianensis Aubl. & Tree & SPSF, 25905 & III, V, VI, XIII & cer, ssf \\
\hline Pachystroma longifolium (Nees) I.M.Johnst. & Tree & & $\mathrm{X}$ & ssf \\
\hline Sapium glandulosum (L.) Morong & Tree & SPSF, 25151 & III, X, XIII & cer, ssf, rp \\
\hline \multicolumn{5}{|l|}{ FABACEAE } \\
\hline Aeschynomene racemosa Vogel & Shrub & & III, $X$ & cer \\
\hline Albizia niopoides (Spruce ex Benth.) Burkart & Tree & & VII, X & $\mathrm{rp}$ \\
\hline Andira anthelmia (Vell.) Benth. & Tree & & III, VI, X & cer, ssf, rp \\
\hline Andira fraxinifolia Benth. & Tree & MBM, 207659 & III, X, XIII & cer \\
\hline Andira humilis Mart. ex Benth. & Tree, Shrub & CGMS, 43980 & VI, XIII & cer, ssf \\
\hline Andira inermis (W.Wright) DC. & Tree & & $\mathrm{I}, \mathrm{X}$ & ssf \\
\hline Andira vermifuga (Mart.) Benth. & Tree & UEC, 44042 & XIII & cer \\
\hline Bauhinia holophylla (Bong.) Steud. & Shrub & SPSF, 17378 & III, X, XIII & cer \\
\hline Bowdichia virgilioides Kunth & Tree & & III, X & cer, ssf \\
\hline Camptosema spectabile (Tul.) Burkart & Climbing & SPSF, 20842 & XIII & cer \\
\hline Canavalia picta Mart. ex Benth. & Climbing & $\mathrm{HRCB}, 52658$ & IV, IX, XIII & cer \\
\hline Cassia ferruginea (Schrad.) Schrad. ex DC. & Tree & UEC, 25224 & I, II, X, XIII & ssf, rp \\
\hline Centrolobium tomentosum Guillem. ex Benth. & Tree & & I, II, V, X & cer, ssf, rp \\
\hline Centrosema sagittatum (Humb. \& Bonpl. ex Willd.) & Climbing & HRCB, 68020; UEC, 170139 & IX, XIII & ssf \\
\hline \multicolumn{5}{|l|}{ Brandegee } \\
\hline Chamaecrista flexuosa (L.) Greene & Shrub & $\mathrm{HRCB}, 52452$ & III, IV, X, XIII & cer \\
\hline Copaifera langsdorffii Desf. & Tree & & I, II, III, V, VI, VII, X & cer, ssf, rp \\
\hline $\begin{array}{l}\text { Dahlstedtia muehlbergiana (Hassl.) M.J.Silva \& } \\
\text { A.M.G. Azevedo }\end{array}$ & Tree & & $\mathrm{I}, \mathrm{X}$ & ssf, rp \\
\hline Dalbergia miscolobium Benth. & Tree & & III, $\mathrm{X}$ & cer \\
\hline Desmodium affine Schltdl. & Shrub & HRCB, 52293 & IV, XIII & $\operatorname{ssf}$ \\
\hline Desmodium distortum (Aubl.) J.F.Macbr. & Shrub & HRCB, 52507 & IV, XIII & cer, ssf \\
\hline Desmodium uncinatum (Jacq.) DC. & Shrub & HRCB, 52391 & IV, XIII & cer, ssf \\
\hline Dimorphandra mollis Benth. & Tree & HRCB, 71802 & III, VI, X, XIII & cer, ssf \\
\hline Dioclea violacea Mart. ex Benth. & Climbing & HRCB, 68021 & IX & ssf \\
\hline Enterolobium contortisiliquum (Vell.) Morong & Tree & & $\mathrm{X}$ & ssf \\
\hline Enterolobium gummiferum (Mart.) J.F.Macbr. & Tree & & III, $X$ & cer \\
\hline Erythrina falcata Benth. & Tree & & III, $\mathrm{X}$ & cer, ssf, rp \\
\hline Holocalyx balansae Micheli & Tree & & II, X & ssf, rp \\
\hline Hymenaea courbaril $\mathrm{L}$. & Tree & HUSC, 11451 & I, X, XIII & ssf, rp \\
\hline Hymenaea stigonocarpa Mart. ex Hayne & Tree & & III, $\mathrm{X}$ & cer \\
\hline Inga edulis Mart. & Tree & & I, X & ssf \\
\hline Inga laurina $(\mathrm{Sw}$.$) Willd.$ & Tree & INPA, 205844 & I, II, X, XIII & ssf, rp \\
\hline
\end{tabular}


Flora of Porto Ferreira State Park

\begin{tabular}{|c|c|c|c|c|}
\hline Inga striata Benth. & Tree & UEC, 49402; UB, 128891 & I, II, V, VI, X, XIII & cer, ssf, rp \\
\hline Inga vera subsp. affinis (DC.) T.D.Penn. & Tree & HRCB, 62049 & VII, X, XIII & $\mathrm{rp}$ \\
\hline Leptolobium dasycarpum Vogel & Tree & SPSF, 24697 & III, VI, X, XIII & cer, ssf \\
\hline Leptolobium elegans Vogel & Tree & UEC, 7418; CGMS, 43984 & III, X, XIII & cer \\
\hline Machaerium aculeatum Raddi & Shrub, Climbing & & $\mathrm{V}, \mathrm{VI}$ & cer, ssf \\
\hline Machaerium acutifolium Vogel & Tree & & III, X & cer \\
\hline Machaerium brasiliense Vogel & $\begin{array}{l}\text { Tree, Shrub, } \\
\text { Climbing }\end{array}$ & & $\mathrm{I}, \mathrm{X}$ & ssf, rp \\
\hline Machaerium stipitatum Vogel & Tree & & III, $\mathrm{X}$ & cer, ssf \\
\hline Machaerium villosum Vogel & Tree & & I, II, III, VI, X & cer, ssf, rp \\
\hline Myroxylon peruiferum L.f. & Tree & & $\mathrm{X}, \mathrm{XIII}$ & ssf, rp \\
\hline Ormosia arborea (Vell.) Harms & Tree & MBM, 189660; IAC, 54532 & I, X, XIII & ssf, rp \\
\hline Peltophorum dubium (Spreng.) Taub. & Tree & SPSF, 49081 & III, VI, VII, X, XIII & cer, ssf, rp \\
\hline Plathymenia reticulata Benth. & Tree & & III, X & cer \\
\hline Platycyamus regnellii Benth. & Tree & & $\mathrm{I}, \mathrm{X}$ & ssf \\
\hline Platypodium elegans Vogel & Tree & CGMS, 43971 & III, VI, X & cer, ssf \\
\hline Pterogyne nitens Tul. & Tree & & II, X & ssf, rp \\
\hline Senna multijuga (Rich.) H.S.Irwin \& Barneby & Tree & & $\mathrm{X}$ & ssf \\
\hline $\begin{array}{l}\text { Senna pendula (Humb.\& Bonpl.ex Willd.) H.S.Irwin } \\
\text { \& Barneby }\end{array}$ & Tree, Shrub & UEC, 44044 & III, X, XIII & cer, ssf \\
\hline Senna rugosa (G.Don) H.S.Irwin \& Barneby & Shrub & SPSF, 25935; HUEFS, 202006 & III, IV, X, XIII & cer, ssf \\
\hline Senna silvestris (Vell.) H.S.Irwin \& Barneby & Tree, Shrub & UEC, 44054 & III, X, XIII & cer, ssf \\
\hline Stryphnodendron polyphyllum Mart. & Tree & & III, $X$ & cer \\
\hline Stylosanthes viscosa (L.) Sw. & Shrub & IAC, 21993 & XIII & cer, ssf, rp \\
\hline Tachigali aurea Tul. & Tree & & III, $\mathrm{X}$ & cer, ssf \\
\hline Zornia latifolia $\mathrm{Sm}$. & Shrub & $\mathrm{APO}, 33$ & IV & cer, ssf \\
\hline \multicolumn{5}{|l|}{ GENTIANACEAE } \\
\hline Voyria aphylla (Jacq.) Pers. & Herb & HRCB, 52305 & IV, XIII & cer, ssf \\
\hline \multicolumn{5}{|l|}{ HYMENOPHYLLACEAE } \\
\hline Crepidomanes pyxidiferum (L.) Dubuisson \& Ebihara & Herb & HRCB, 71765 & XIII & ssf \\
\hline \multicolumn{5}{|l|}{ IRIDACEAE } \\
\hline Cipura paludosa Aubl. & Herb & GMM,798 & XIII & cer \\
\hline \multicolumn{5}{|l|}{ LACISTEMATACEAE } \\
\hline Lacistema hasslerianum Chodat & Shrub & SPSF, 49083; UEC, 25118 & I, III, V, VI, VII, X, XIII & cer, ssf, rp \\
\hline
\end{tabular}




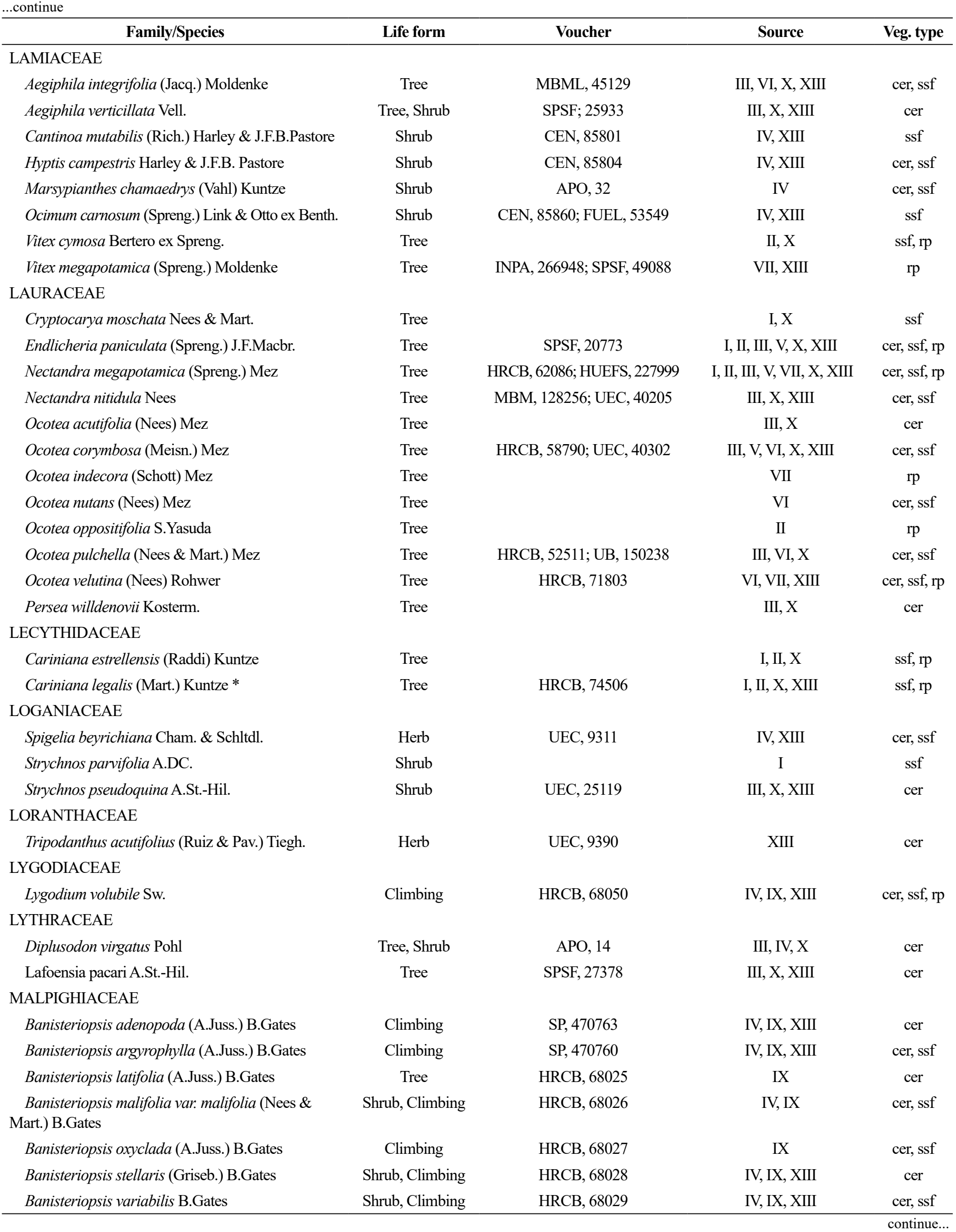




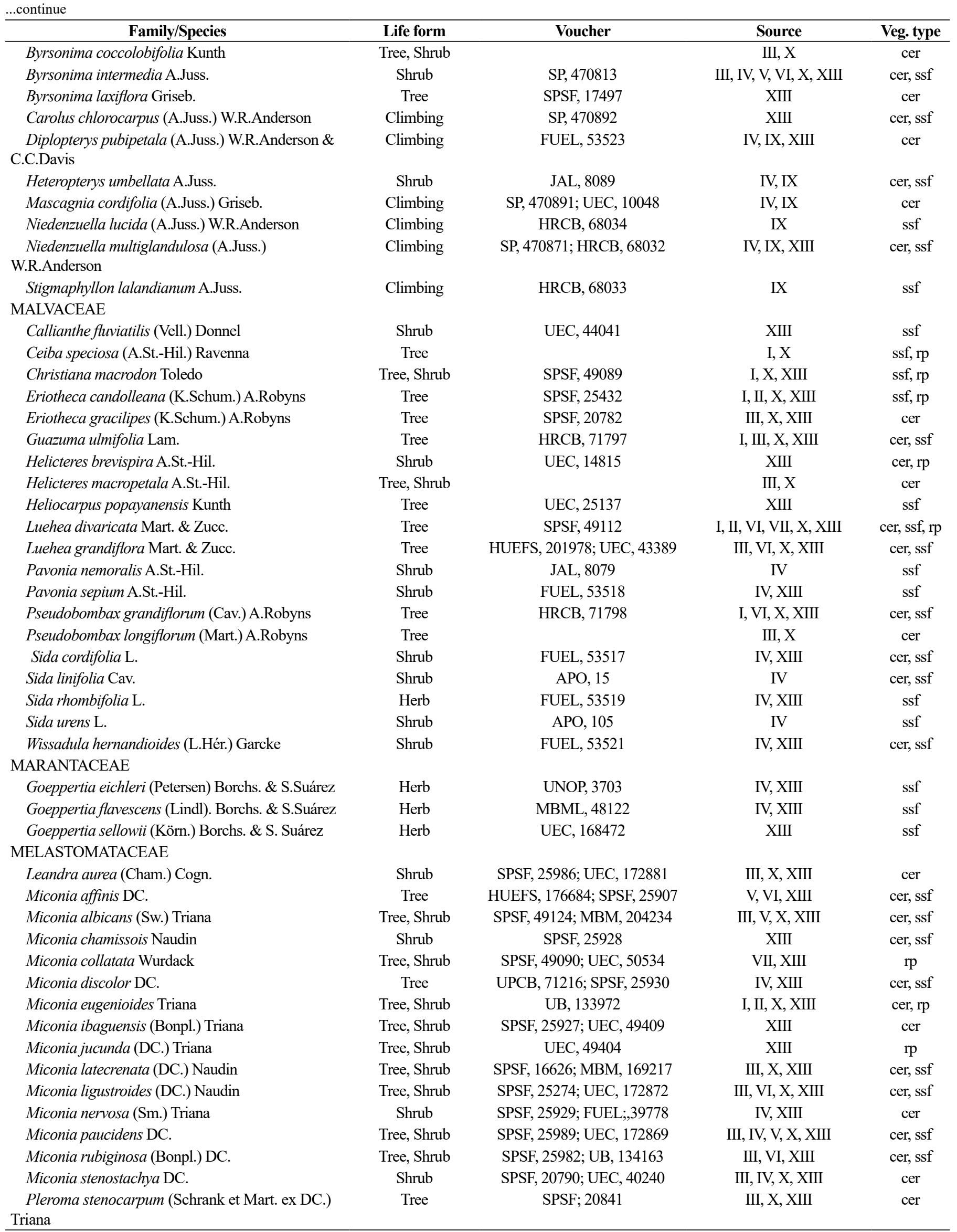




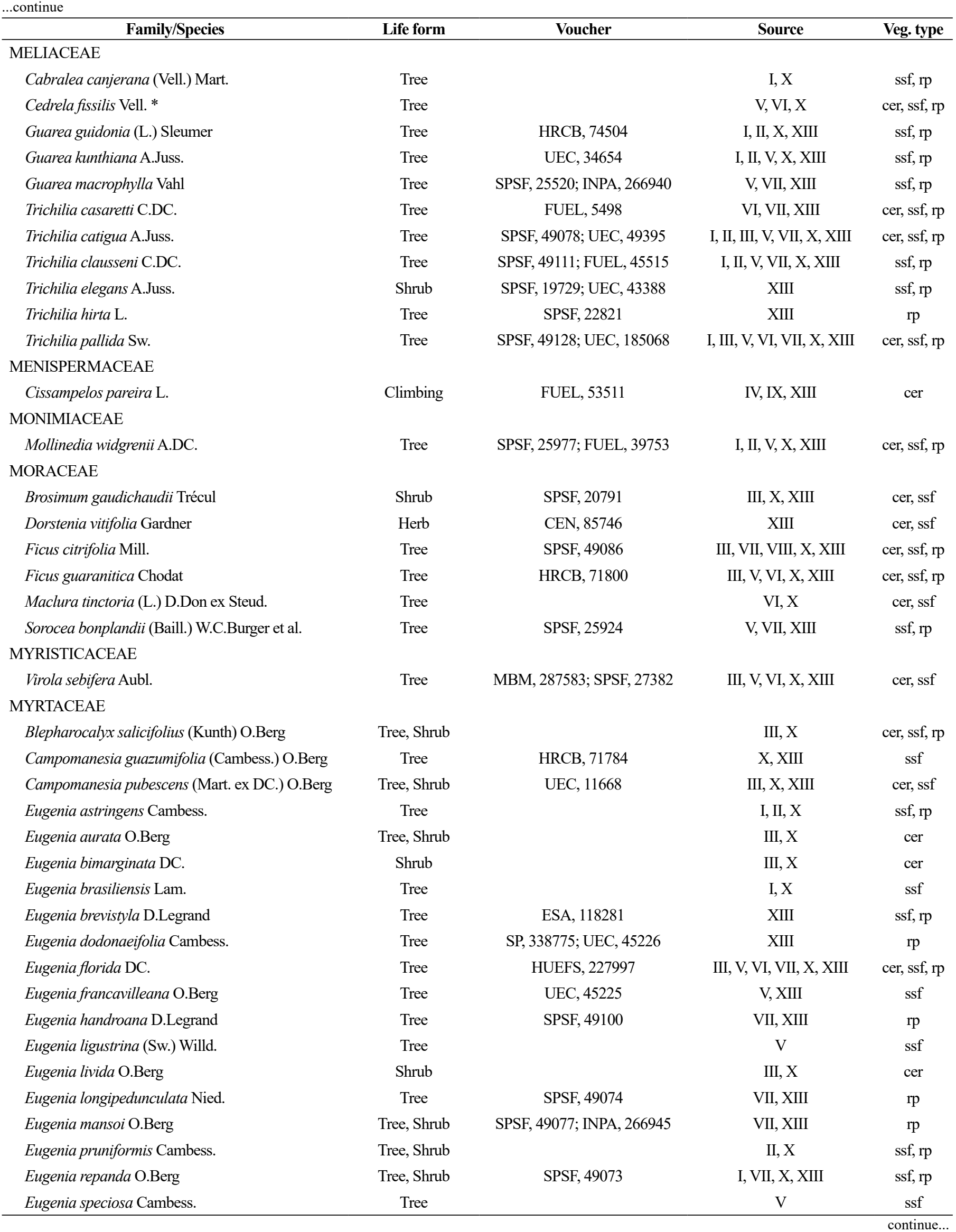


Flora of Porto Ferreira State Park

\begin{tabular}{|c|c|c|c|c|}
\hline Eugenia subterminalis DC. & Tree & & $\mathrm{X}$ & ssf, rp \\
\hline Eugenia verticillata (Vell.) Angely & Shrub & & II, $X$ & ssf, rp \\
\hline Myrcia albotomentosa DC. & Shrub & SPSF, 25964 & III, X, XIII & cer \\
\hline Myrcia bella Cambess. & Shrub & & III, VI, X & cer, ssf \\
\hline Myrcia guianensis (Aubl.) DC. & Tree & SPSF, 25406 & III, VI, X, XIII & cer, ssf \\
\hline Myrcia hebepetala DC. & Tree & & II, III, X & cer, rp \\
\hline Myrcia lasiantha DC. & Shrub & & III, X & cer \\
\hline Myrcia multiflora (Lam.) DC. & Tree, Shrub & SPSF, 10654; UEC, 40239 & I, II, III, V, X, XIII & cer, ssf, rp \\
\hline Myrcia venulosa DC. & Tree & SPSF, 25984; UEC, 12123 & VI, XIII & cer, ssf \\
\hline Myrcianthes pungens (O.Berg) D.Legrand & Tree & & VII & $\mathrm{rp}$ \\
\hline Myrciaria floribunda (H.West ex Willd.) O.Berg & Tree & $\mathrm{HRCB}, 74505$ & II, X, XIII & ssf, rp \\
\hline Myrciaria tenella (DC.) O.Berg & Tree & IAC, 54946 & VII, XIII & $\mathrm{rp}$ \\
\hline Plinia cauliflora (Mart.) Kausel & Tree & & $\mathrm{I}, \mathrm{X}$ & ssf, rp \\
\hline Psidium grandifolium Mart. ex DC. & Shrub & SPSF, 25268 & XIII & cer \\
\hline Psidium guineense $\mathrm{Sw}$. & Tree, Shrub & UEC, 12340 & III, X, XIII & cer, ssf \\
\hline Psidium rufum Mart. ex DC. & Tree & SPSF, 20853 & III, VI, X, XIII & cer, ssf \\
\hline Siphoneugena densiflora O.Berg & Tree & & VII & $\mathrm{rp}$ \\
\hline Ouratea spectabilis (Mart.) Engl. & Tree & & III, X & cer \\
\hline \multicolumn{5}{|l|}{ OPILIACEAE } \\
\hline Agonandra excelsa Griseb. & Tree, Shrub & & $\mathrm{V}$ & ssf \\
\hline \multicolumn{5}{|l|}{ ORCHIDACEAE } \\
\hline Acianthera macuconensis (Barb.Rodr.) F.Barros & Herb & HRCB, 62376 & VIII & ssf \\
\hline Acianthera pubescens (Lindl.) Pridgeon \& M.W.Chase & Herb & HRCB, 62034 & IV, VIII & ssf, rp \\
\hline Acianthera recurva (Lindl.) Pridgeon \& M.W.Chase & Herb & $\mathrm{HRCB}, 62390$ & VIII & $\mathrm{rp}$ \\
\hline $\begin{array}{l}\text { Acianthera saundersiana (Rchb.f.) Pridgeon \& } \\
\text { M.W.Chase }\end{array}$ & Herb & $\mathrm{HRCB}, 62015$ & VIII & $\mathrm{rp}$ \\
\hline Anathallis obovata (Lindl.) Pridgeon \& M.W.Chase & Herb & HRCB, 62907 & VIII & $\mathrm{rp}$ \\
\hline Aspidogyne argentea (Vell.) Garay & Herb & HRCB, 62022 & XIII & $\mathrm{rp}$ \\
\hline Bulbophyllum epiphytum Barb.Rodr. & Herb & HRCB, 62378 & VIII & ssf \\
\hline Bulbophyllum tripetalum Lindl. & Herb & GMM, 208 & VIII & $\mathrm{rp}$ \\
\hline Campylocentrum crassirhizum Hoehne & Herb & HRCB, 62038 & VIII & cer, ssf, rp \\
\hline Campylocentrum grisebachii Cogn. & Herb & $\mathrm{HRCB}, 62373$ & VIII & $\mathrm{rp}$ \\
\hline Campylocentrum sellowii (Rchb.f.) Rolfe & Herb & & VIII & $\mathrm{rp}$ \\
\hline Catasetum fimbriatum (C.Morren) Lindl. & Herb & HRCB, 62037 & VIII & cer, ssf \\
\hline
\end{tabular}




\begin{tabular}{|c|c|c|c|c|}
\hline Cattleya loddigesii Lindl. & Herb & $\mathrm{HRCB}, 63646$ & VIII & $\mathrm{rp}$ \\
\hline Cattleya walkeriana Gardner* & Herb & HRCB, 62908 & VIII & $\mathrm{rp}$ \\
\hline Christensonella neowiedii (Rchb.f.) S.Koehler & Herb & HRCB, 62014 & VIII & ssf \\
\hline Cyclopogon elatus (Sw.) Schltr. & Herb & HRCB, 62013 & XIII & $\mathrm{rp}$ \\
\hline Dryadella aviceps (Rchb.f.) Luer & Herb & HRCB, 62036 & VIII & ssf, rp \\
\hline Encyclia patens Hook. & Herb & & VIII & $\mathrm{rp}$ \\
\hline Epidendrum latilabrum Lindl. & Herb & HUFU, 72366 & VIII & $\mathrm{rp}$ \\
\hline Epidendrum rigidum Jacq. & Herb & HUFU, 72316 & VIII, XIII & $\mathrm{rp}$ \\
\hline Isochilus linearis (Jacq.) R.Br. & Herb & HRCB, 62384 & VIII & ssf, rp \\
\hline Laelia gloriosa (Rchb.f.) L.O.Williams & Herb & HRCB, 63669 & VIII & $\mathrm{rp}$ \\
\hline Liparis nervosa (Thumb.) Lindl. & Herb & $\mathrm{HRCB}, 62372$ & XIII & $\mathrm{rp}$ \\
\hline Maxillaria marginata (Lindl.) Fenzl & Herb & HRCB, 63317 & VIII & ssf, rp \\
\hline Mesadenella cuspidata (Lindl.) Garay & Herb & UEC, 160185 & IV, XIII & $\mathrm{rp}$ \\
\hline Miltonia flavescens (Lindl.) Lindl. & Herb & HUFU, 72315 & VIII, XIII & ssf, rp \\
\hline Notylia hemitricha Barb.Rodr. & Herb & $\mathrm{HRCB}, 62377$ & VIII & ssf \\
\hline Octomeria warmingii Rchb.f. & Herb & GMM, 181 & VIII & ssf, rp \\
\hline Pelexia laxa (Poepp. \& Endl.) Lindl. & Herb & HRCB, 62021 & XIII & $\mathrm{rp}$ \\
\hline \multicolumn{5}{|l|}{ N.H.Williams } \\
\hline Oxalis physocalyx Zucc. ex Progel & Shrub & UEC, 45224 & III, X, XIII & cer \\
\hline \multicolumn{5}{|l|}{ PASSIFLORACEAE } \\
\hline Passiflora alata Curtis & Climbing & UNOP, 8279 & IV, IX, XIII & $\operatorname{ssf}$ \\
\hline Passiflora miersii Mast. & Climbing & HRCB, 71788 & IV, IX, XIII & cer \\
\hline Passiflora pohlii Mast. & Climbing & SPSF, 20847; MO, 100510557 & XIII & cer, ssf \\
\hline Passiflora suberosa L. & Climbing & APO, 02 & IV, IX & cer \\
\hline Passiflora tenuifila Killip & Climbing & UEC, 40164; HURB, 2457 & XIII & ssf \\
\hline \multicolumn{5}{|l|}{ PERACEAE } \\
\hline Pera glabrata (Schott) Poepp. ex Baill. & Tree & SPSF, 20846 & III, V, VI, X, XIII & cer, ssf, rp \\
\hline \multicolumn{5}{|l|}{ PHYLLANTACEAE } \\
\hline Phyllanthus tenellus Roxb. & Herb & CEN, 85800 & IV, XIII & ssf \\
\hline Savia dictyocarpa Müll.Arg. & Tree & & $\mathrm{I}, \mathrm{X}$ & ssf, rp \\
\hline \multicolumn{5}{|l|}{ PHYTOLACCACEAE } \\
\hline Gallesia integrifolia (Spreng.) Harms & Tree & & $\mathrm{X}$ & ssf \\
\hline Phytolacca dioica $\mathrm{L}$. & Tree & SPSF, 20851 & XIII & cer \\
\hline \multicolumn{5}{|l|}{ PICRAMNIACEAE } \\
\hline Picramnia ramiflora Planch. & Tree & SPSF, 25996; UEC, 25467 & II, X, XIII & ssf, rp \\
\hline Picramnia sellowii Planch. & Tree, Shrub & SPSF, 49091; INPA, 266939 & VII, XIII & $\mathrm{rp}$ \\
\hline
\end{tabular}




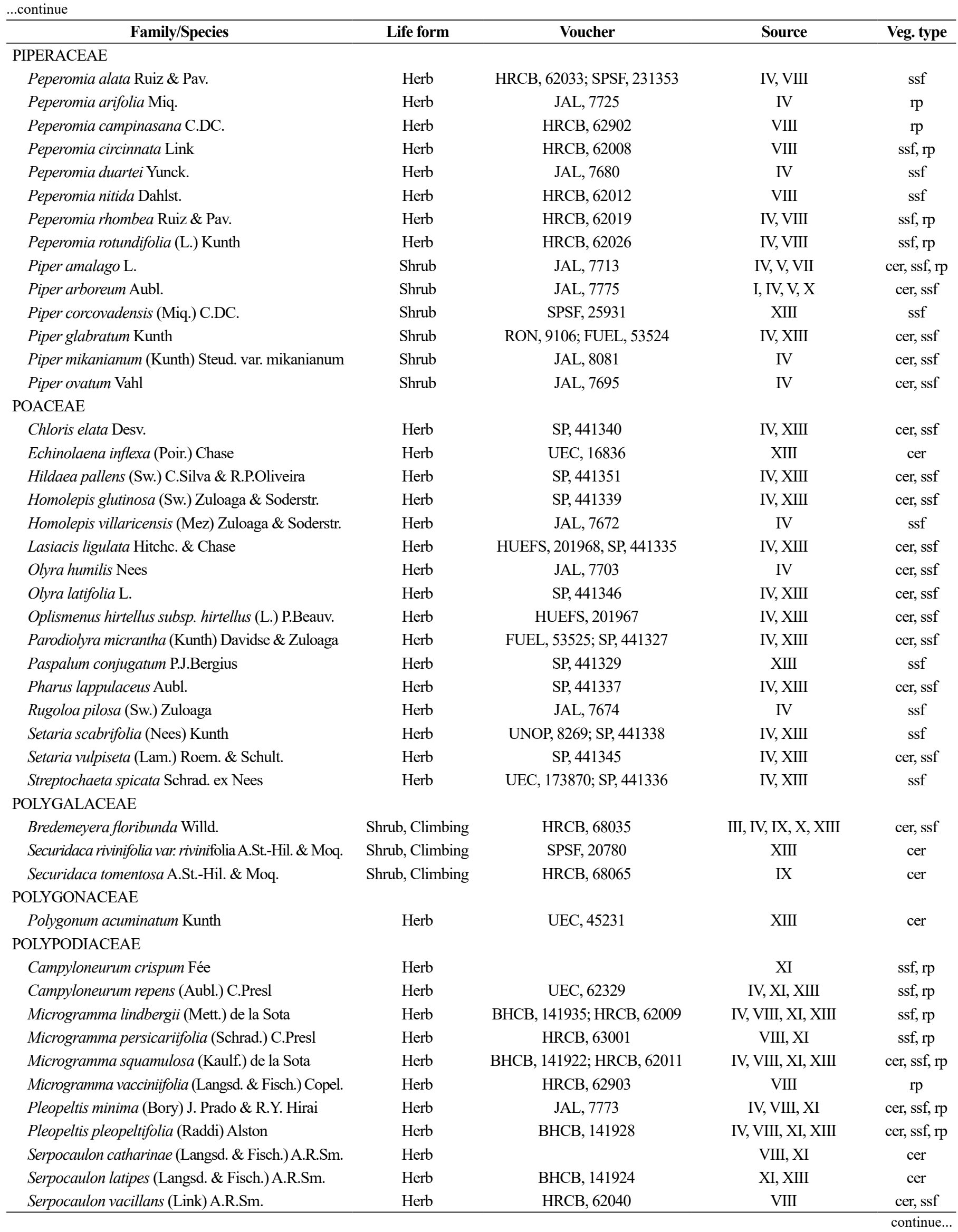




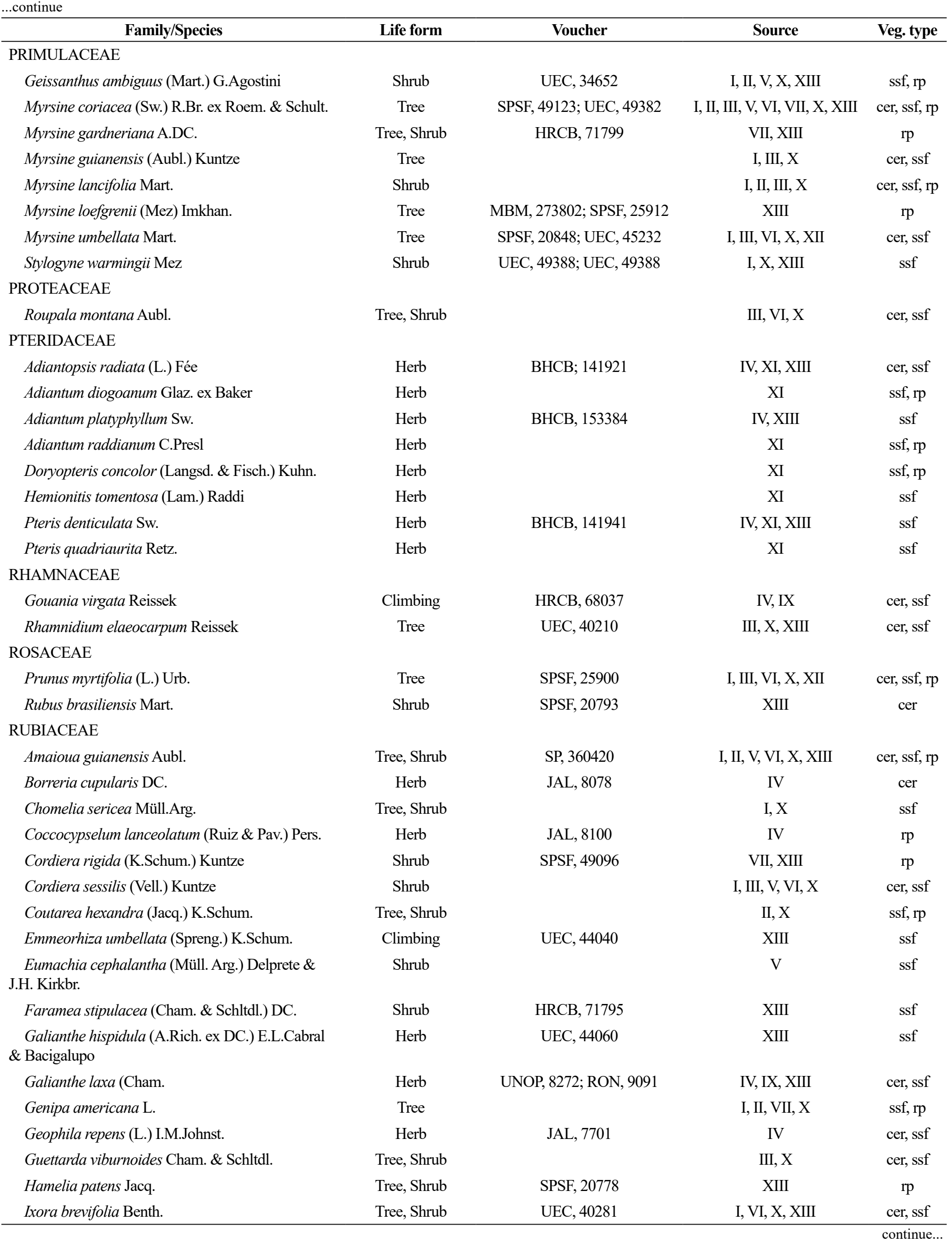


Flora of Porto Ferreira State Park

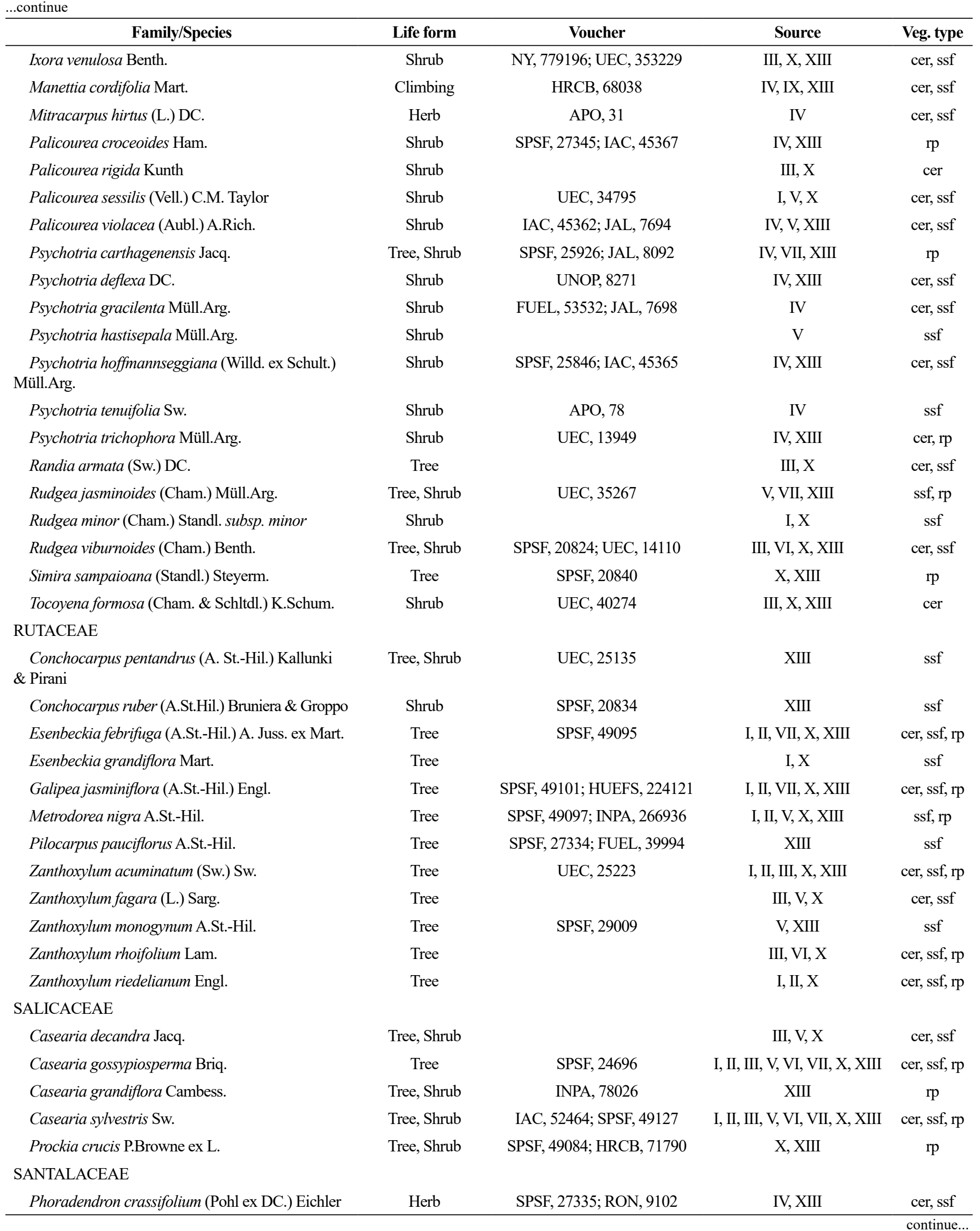




\begin{tabular}{|c|c|c|c|c|}
\hline Allophylus racemosus $\mathrm{Sw}$. & Shrub & $\mathrm{HRCB}, 71792$ & XIII & ssf \\
\hline Allophylus semidentatus (Miq.) Radlk. & Tree & & I, II, VI, X & cer, ssf, rp \\
\hline Cardiospermum grandiflorum Sw. & Climbing & HRCB, 68039 & IX & ssf \\
\hline Cupania vernalis Cambess. & Tree & SPSF, 17376; UEC, 44049 & III, V, VI, X, XIII & cer, ssf, rp \\
\hline Matayba elaeagnoides Radlk. & Tree & SPSF, 49079; INPA, 204885 & V, VII, XIII & cer, ssf, rp \\
\hline Matayba guianensis Aubl. & Tree & SPSF, 17375 & I, II, III, X, XIII & cer, ssf, rp \\
\hline Paullinia rhomboidea Radlk. & Shrub, Climbing & RON, 9096 & IV, IX, XIII & cer, ssf, rp \\
\hline Serjania communis Cambess. & Climbing & FUEL, 53531; UNOP, 8275 & IV, IX, XIII & cer \\
\hline Serjania meridionalis Cambess. & Climbing & UNOP, 8274; FUEL, 53543 & IV, IX, XIII & ssf \\
\hline Serjania paradoxa Radlk. & Climbing & RON, 9093 & IV, IX, XIII & cer \\
\hline Serjania pinnatifolia Radlk. & Climbing & HRCB, 68055; FUEL, 53513 & IV, IX, XIII & cer, ssf \\
\hline Serjania reticulata Cambess. & Climbing & APO, 48 & IV, IX & cer, ssf \\
\hline Urvillea laevis Radlk. & Climbing & RON, 9092; UNOP, 8273 & IV, IX, XIII & cer, ssf \\
\hline \multicolumn{5}{|l|}{ SAPOTACEAE } \\
\hline $\begin{array}{l}\text { Chrysophyllum gonocarpum (Mart. \& Eichler ex Miq.) } \\
\text { Engl. }\end{array}$ & Tree & SPSF, 49087 & I, II, VII, X, XIII & ssf, rp \\
\hline Chrysophyllum marginatum (Hook. \& Arn.) Radlk. & Tree, Shrub & SPSF, 20833 & III, V, VI, X, XIII & cer, ssf \\
\hline Pouteria ramiflora (Mart.) Radlk. & Tree, Shrub & & III, $\mathrm{X}$ & cer, ssf \\
\hline \multicolumn{5}{|l|}{ SOLANACEAE } \\
\hline Capsicum flexuosum Sendtn. & Shrub & SPSF; 25610 & XIII & ssf \\
\hline Cestrum mariquitense Kunth & Shrub & SPSF, 25845 & XIII & cer \\
\hline Cestrum pedicellatum Sendtn. & Shrub & UEC, 44039 & III, X, XIII & cer \\
\hline Solanum acerifolium Dunal & Shrub & BHCB, 141929 & IV, XIII & cer, ssf \\
\hline Solanum americanum Mill. & Herb & JAL, 7664 & IV & ssf \\
\hline Solanum campaniforme Roem. \& Schult. & Shrub & UEC, 44059 & XIII & ssf \\
\hline Solanum granulosoleprosum Dunal & Tree & SPSF, 20786; FUEL, 31988 & XIII & cer \\
\hline Solanum lantana Sendtn. & Shrub & SPSF, 20827; HUEFS, 201965 & IV, XIII & cer, ssf \\
\hline Solanum lycocarpum A.St.-Hil. & Tree, Shrub & SPSF, 27344; BHCB, 141930 & III, IV, X, XIII & cer, ssf \\
\hline Solanum paniculatum $\mathrm{L}$. & Shrub & SPSF, 25899 & III, X, XIII & cer \\
\hline Solanum swartzianum Roem. \& Schult. & Tree & JAL, 7687 & I, III, IV, V, X & cer, ssf \\
\hline Solanum uncinellum Lindl. & Climbing & HRCB, 71796 & XIII & ssf \\
\hline \multicolumn{5}{|l|}{ STYRACACEAE } \\
\hline Styrax acuminatus Pohl & Tree & & $\mathrm{V}, \mathrm{VI}$ & cer, ssf \\
\hline Styrax camporum Pohl & Tree & UEC, 40658; IAC, 54616 & III, VI, X, XIII & cer, ssf \\
\hline Styrax ferrugineus Nees \& Mart. & Tree, Shrub & FUEL, 53529; RON, 9104 & VI, XIII & cer, ssf \\
\hline Styrax pohlii A.DC. & Tree, Shrub & & III, X & cer, ssf \\
\hline
\end{tabular}




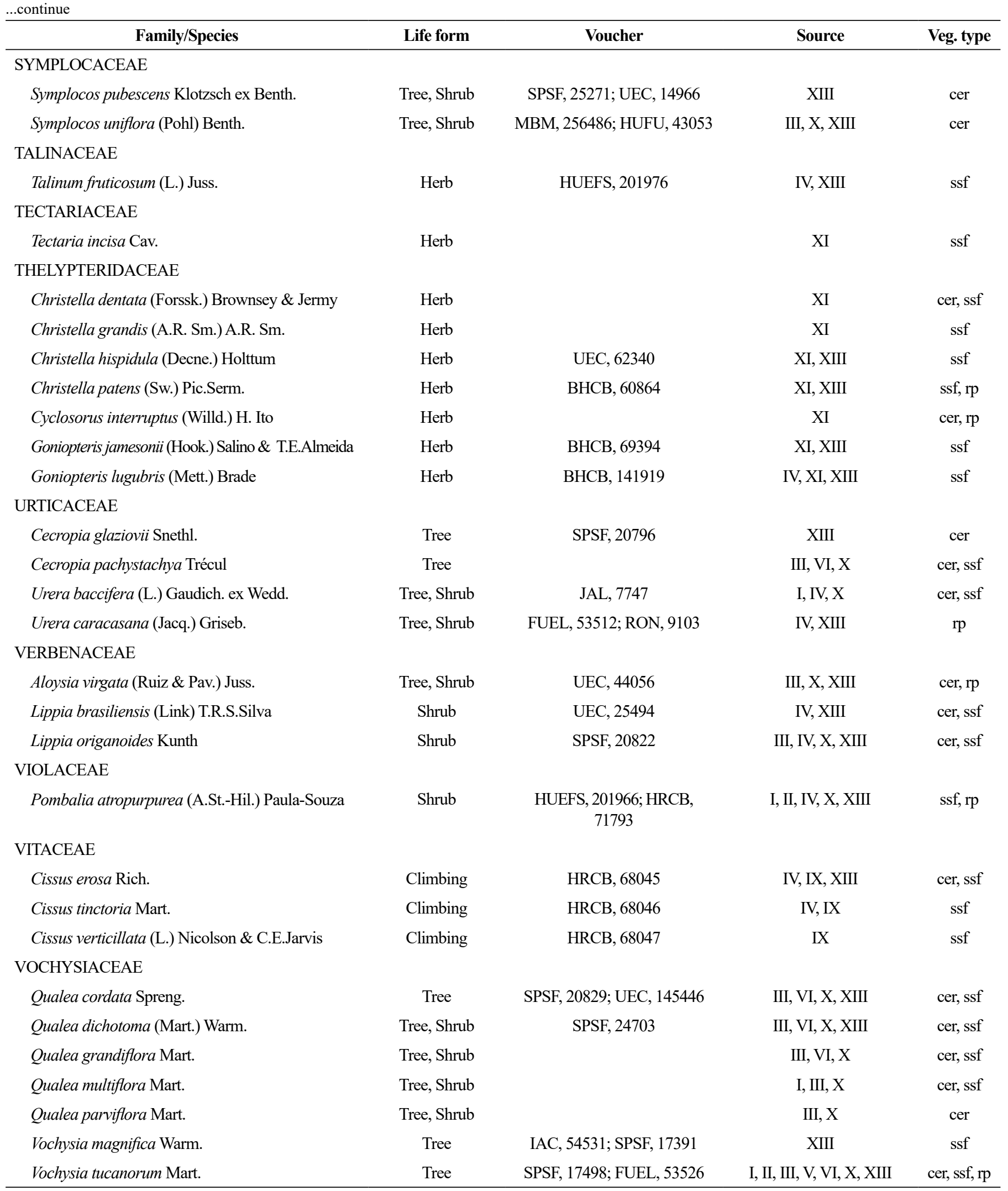

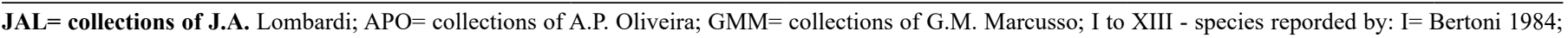
II= Bertoni \& Martins 1987; III= Bertoni et al. 2001; IV= Oliveira 2012; V= Osaco 2012; VI= Sabino 2013; VII= Konopczyk 2014; VIII= Marcusso et al. 2016; IX= Vargas et al. 2018; X= São Paulo 2003; XI= Colli et al. 2003; XII= Dickfeldt et al. 2013; XIII= present study; Asterisks indicate threatened species according to the "Livro Vermelho da Flora do Brasil" by CNCFlora (Martinelli \& Moraes 2013) and the list of threatened flora of São Paulo State (SMA Resolution 2016); Veg. type: vegetation types of occurrence; cer= cerradão; ssf: seasonal semideciduous forest; rp: riparian forest. 


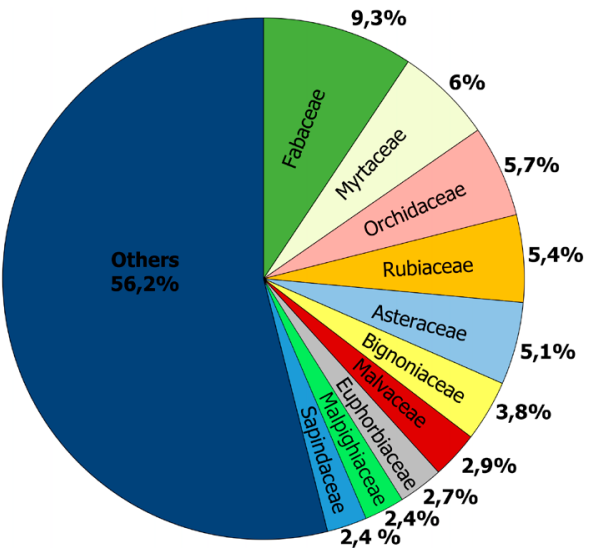

Families (richness)

$\square$ Fabaceae (64)

$\square$ Myrtaceae (41)

$\square$ Orchidaceae (39)

$\square$ Rubiaceae (37)

$\square$ Asteraceae (35)

$\square$ Bignoniaceae (26)

- Malvaceae (20)

$\square$ Euphorbiaceae (19)

$\square$ Malpighiaceae (17)

$\square$ Sapindaceae (17)

a Others (369)

Figure 3. The most species-rich families in the Porto Ferreira State Park, Porto Ferreira, São Paulo state, southeastern Brazil.

The arboreal habit was the predominant life form in the PFSP $(41.8 \%)$, followed by herbs $(25.7 \%)$, shrubs/subshrubs (17.9\%) and climbing plants (14.7\%) (Figure 4). The results listed 84 species (12.2\%) with two or more life forms. Concerning all life forms, 9.6\%, 18.8\% and $18.5 \%$ of the species are exclusive to RP, CER and SSF, respectively. The number of exclusive species for the CER is mainly related to the high number of trees species. However, herbs represented $40 \%$ and $50 \%$ of the exclusive species found in the SSF and RP, respectively.

The number of species compiled for the lists of the floristic similarity comparison was 2745, of which $1420(51.7 \%)$ are exclusively from a single area (Appendix 2). Only 43 species (1.5\%) occurred in $50 \%$ or more of the lists, and no species were recorded for all areas (Appendix 3). The dendrogram showed a high coefficient of cophenetic correlation of 0.82 . The greater the distance was between areas, the greater the floristic dissimilarities among them. The cluster analysis showed the segregation of three major floristic groups (Figure 5). The first group (1) clustered an ecotone area and two RP (one of which belongs to the same survey as the ecotone); the second group (2) categorized the studies carried out in Cerrado vegetation types; and the third group (3) all vegetation types of the PFSP and areas under SSF formation.

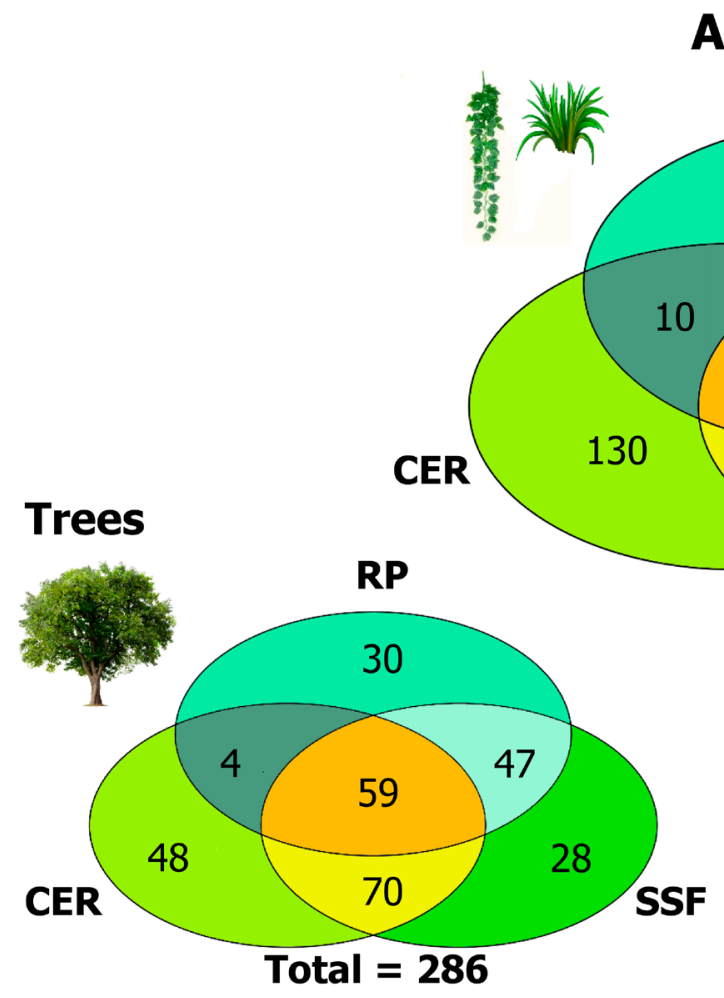

All life forms
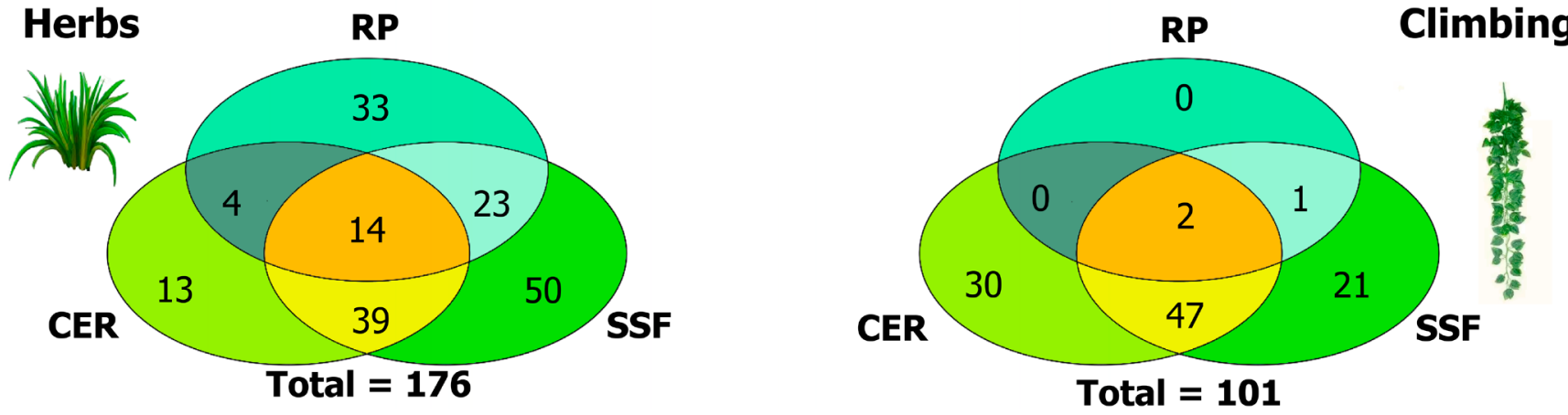

Figure 4. Venn diagrams for vascular plants recorded in Porto Ferreira State Park, Porto Ferreira, São Paulo State, southeastern Brazil. RP: riparian forest, SSF: seasonal semidecidual forest; CER: cerradão. 


\section{Floristic similarity}

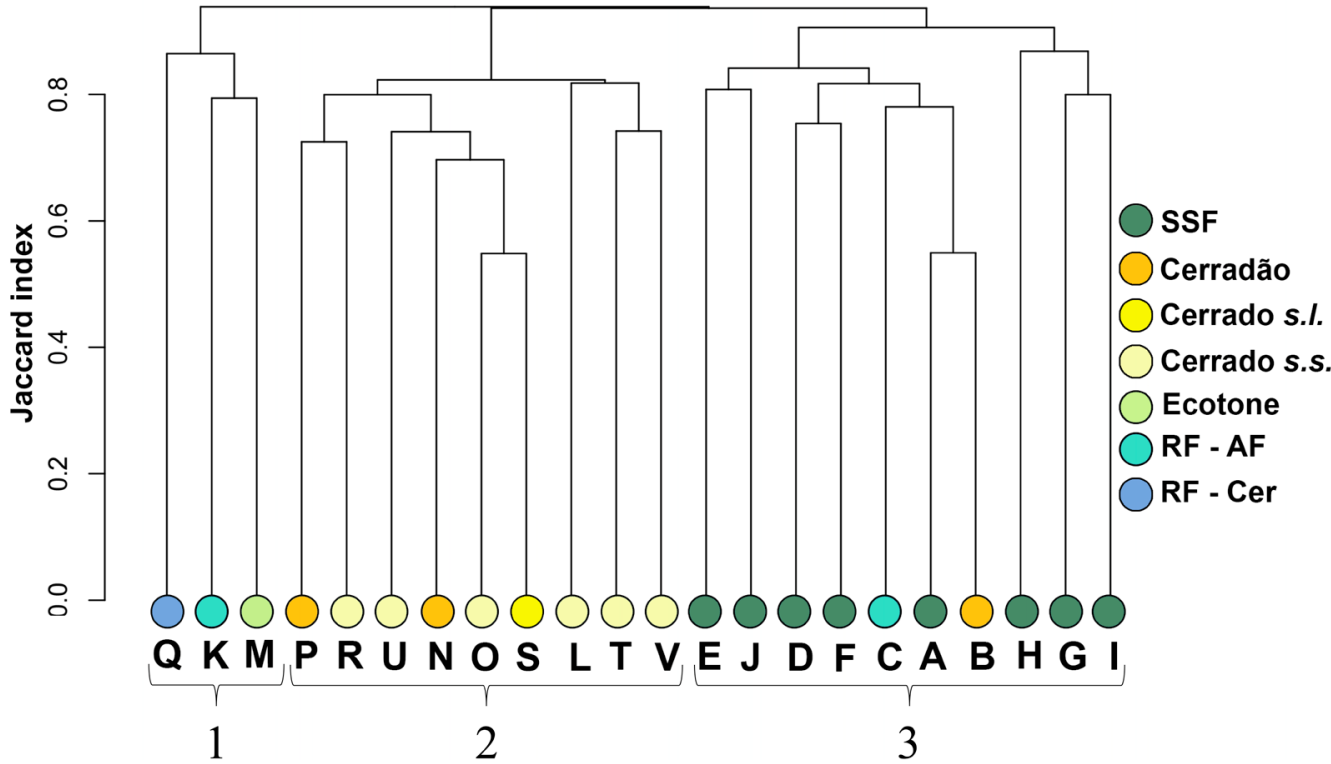

Figure 5. Cluster analysis (UPGMA) using Jaccard index among fifteen studies carried out in Cerrado, SSF and riparian forest vegetation. Areas (identified by letters A-V) are given in Table 1. SSF: seasonal semidecidual forest; Cerrado s.l.: Cerrado sensu lato; Cerrado s.s.: Cerrado sensu stricto; RF-AF: riparian forest under Atlantic Forest ecoregion; RF-Cer: riparian forest under Cerrado ecoregion.

\section{Discussion}

The comprehensive checklist of the PFSP allowed us to show that the vascular flora in this ecotonal area is composed of a mixture of elements of both the Atlantic Forest and Cerrado biogeographical provinces. The soil features are a possible explanation for the different vegetation types, and variations on a small scale. The overall richness is impressive, considering the small size of park; however, the number of species of each vegetation type recorded is in accordance with previous studies in these vegetation types. An Atlantic Forest similarity was found for the PFSP flora as a whole. The same relationship was found considering the CER and SSF floras independently, demonstrating the strong influence of Atlantic Forest on the area. Perhaps the geographical location of the PFSP, which reaches both biogeographical provinces in the eastern region, could be a reasonable explanation for these results.

The species richness of the PFSP was higher than that found by Cielo-Filho et al. (2015) - with 265 species -, the only vascular flora surveyed in an ecotonal site among Cerrado and Atlantic Forest in southeastern Brazil. The overwhelming number of species recorded for the PFSP vascular flora is ranked fourth among studies conducted under seasonal formations, behind only those of Lombardi \& Gonçalves (2000), Lombardi et al. (2012) and Forzza et al. (2014). Species richness tends to peak in ecotonal areas (Kark \& van Rensburg, 2006), likely because transitional areas hold species from two or more neighboring communities, and due to high spatial heterogeneity (Kark 2012). Furthermore, along with the increase in botanical collection in protected areas, there is an appeal to enhance the importance of these areas to protect threatened species (Colli-Silva et al., 2019). In this sense, we found eight threatened species at regional and national levels. Thus, the ecotonal condition of the PFSP between two biogeographical provinces may contribute to increase the species richness, also being home to important threatened species.
Studies have been emphasizing the need for the expansion of botanical collection in protected areas (Colli-Silva et al. 2016, 2019). The addition of more than $65 \%$ of the species number since the last PFSP floristic list (São Paulo, 2003) is significant, showing the importance of continuous research, and fieldwork, to update the knowledge about the flora. Since the Colli-Silva et al. study of 2016, which recorded 292 species for the PFSP, 235 vouchers have been added to the PFSP, most likely due to the digitization of the collections, later available in digital platforms (CRIA, 2019), and to the addition of our own collections. Moreover, notably there are species that we found in the field, such as Cissus serroniana (Glaz.) Lombardi (Vitaceae), which had never before been recorded fertile, and, therefore, were not included in the checklist. Even though the PFSP has a significant number of studies concerning its flora, we predict that further collections will increase the number of species in this protected area.

Considering each vegetation type of the PFSP, the richness we found in the SSF averages roughly with that found in other studies (Corrêa et al., 2018; Cielo-Filho et al., 2015; Forzza et al., 2014; Pifano et al., 2013; Rossetto \& Vieira, 2013; Lombardi et al., 2012; Guaratini et al., 2008; Lombardi \& Gonçalves, 2000). In the CER, the number of species recorded in our study is higher than other Cerrado surveys (Cavassan \& Weiser, 2015; Cielo-Filho et al. 2015; Carvalho et al., 2010; Ishara et al., 2008; Batalha \& Mantovani, 2001; Durigan et al., 1999; Batalha et al., 1997). As for the RP, there are no studies of vascular flora carried out specifically on this vegetation type, but rather inevitably included as a part of a larger work, such as those of Durigan et al. (1999) and Cielo-Filho et al. (2015), making comparison difficult.

The most species-rich families found in our study are also the richest ones for the Cerrado as a whole (Souza, et al. 2018), and seasonal forests of the Atlantic Forest (Souza, et al., 2019a). Orchidaceae, Fabaceae, Asteraceae and Myrtaceae are, respectively, the most diverse families in the Atlantic Forest (BFG, 2015). 
Thus, Fabaceae, Asteraceae and Orchidaceae constitute the three richest families in the Cerrado (Mendonça et al. 2008). The richest genera found in the PFSP - Eugenia, Micona and Myrcia - are ranked as the genera with the highest number of tree species in the Cerrado and the Atlantic Forest, under semideciduous formation (Oliveira-Filho \& Fontes 2000). Considering Brazilian flora as a whole, Eugenia, Miconia, Myrcia, Psychotria, Solanum and Peperomia are also classified among the 30 most diverse Angiosperm genera (BFG, 2015).

Generalist species, establishing and thriving both in the Cerrado and in the Atlantic Forest, generally become very abundant in transition areas between these two biogeographical provinces (Durigan et al. 2012, Morrone, 2014, 2017). All species classified as generalists by Durigan et al. (2012) were found in the PFSP flora [e.g. Copaifera langsdorffii Desf., Cordia trichotoma (Vell.) Arráb. ex Steud, Mabea fistulifera Mart., Ocotea corymbosa (Meisn.) Mez, Platypodium elegans Vogel, Senegalia polyphyla (DC.) Britton \& Rose, Terminalia glabrescens Mart. and Vochysia tucanorum Mart.]. Durigan et al. (2012) also pointed out 20 typical species of SSF, of which 15 were reported in the PFSP flora (e.g. Aspidosperma polyneuron Müll.Arg., Cedrela fissilis Vell., Metrodorea nigra A.St.-Hil. and Guarea kunthiana A. Juss.). In a study encompassing most of the Cerrado woody vegetation, Ratter et al. (2003) found 38 species with the highest percentage of occurrence, of which 26 (68.4\%) occur in PFSP CER (e.g. Qualea grandiflora Mart., Qualea parviflora Mart., Bowdichia virgilioides Kunth, Dimorphandra mollis Benth., Lafoensia pacari A.St.-Hil., Connarus suberosus Planch. and Hymenaea stigonocarpa Mart. ex Hayn). These results emphasize the PFSP as a species reservatory of several biogeographical entities, reinforcing its role in conservation.

The trees were the most species-rich life forms in the PFSP, following the patterns of forest vegetation types (IBGE, 2012; Forzza et al., 2014). Regarding the shared species among the vegetation types, trees perform a major contribution to the exclusive species of the CER. The different edaphic physicochemical features among the CER and SSF are the main causes of their differences, even in small scales (Gottsberger \& Silberbauer-Gottsberger, 2006; Pinheiro et al. 2009). Edaphic factors, such as water availability in the soil, and soil composition, may play a role in vegetation type differentiation (Haridasan 1992). The heterogeneity of the soil, on a small-scale, can also act as a plant community ecological driver, increasing diversity and promoting structural changes (Souza et al., 2019b). In a study carried out on the PFSP soil and its relationship to the vegetation, Rossi et al. (2005) found several types of soil. The authors also found an association between CER, SSF and RP vegetation and dystrophic oxisols, eutrophic argisols and dystrophic fluvial neosols, respectively.

A substantial number of herbs species were found in the PFSP, including the epiphytic and terricolous habit. The impressive importance of the herbs in the PFSP is most likely due to the contribution of the Cerrado elements; usually the number recorded in the AF is lower than the one we recorded here (Vieira et al., 2015). An explanation for this may be the ecotonal feature of the PFSP, and the high number of shared species between the CER and SSF. Thus, even in the Cerrado forestry vegetation type, the herbaceous layers must be considered as an important synusia. On the other hand, the high number of exclusive herbaceous species in the RP must be attributed to the microclimatic conditions propitious to the epiphytes' development (Marcusso et al., 2016).
The number of species of climbing plants in the present study was lower than that found by Vargas et al. (2018), because we considered only taxa identified at the taxonomic level of species and, occasionally, used the life form classification according to the Flora do Brasil (2020). This classification does not always reflect Vargas' et al. (2018) interpretations.

We found an outstanding heterogeneity among the floristic surveys analyzed, in which very few species occurred in $50 \%$ or more of the areas, and no species occurred in all areas. This may be due to the addition of surveys from different vegetation types belonging to the Cerrado and the Atlantic Forest. Floristic comparisons are usually made between vegetation types within the same biogeographical provinces (e.g. Carvalho et al. 2010; Ishara et al., 2008; Guaratini et al., 2008; Pifano et al., 2013). Here, including areas of both Cerrado and Atlantic Forest, we found that the areas with the same vegetation type are more inclined to be floristically similar. However, in ecotones, areas with different vegetation types in the same location tend to share more species with each other than areas in which the same vegetation type occurs at a distance from one another. These results have been reported for several studies (e.g. Oliveira-Filho \& Fontes, 2000; Oliveira-Filho et al., 2015; Dryflor et al., 2016), contrary to the general biogeographic hypothesis that similar species would be shared between regions due to the restriction of their dispersion capacity (Lieberman, 2003). Therefore, the fact that the CER of the PFSP joined in the SSF group might be due to the high spatial proximity correlation between them, suggesting a strong relationship with this matrix (Pinheiro \& Monteiro, 2008; Passos et al., 2018), with the SSF exerting more influence over the CER flora.

The PFSP harbours eight threatened species, and a huge number of vascular plant species. We also found a greater floristic similarity within the PFSP, and studies carried out in the Atlantic Forest. There is a lack of floristic studies that include all life forms conducted in ecotonal areas in southeastern Brazil. The reason for this, we believe, is due to the difficulty in recognizing an ecotone, and the negligence on supporting descriptive studies. This is an unprecedented floristic list recorded for the PFSP. The overwhelming diversity and the pool of species found, highlights this remnant as an important protected area. Results shown here can be used as a floristic reference for future conservation measures, and restorative actions in areas under ecotonal influences of the Atlantic Forest and the Cerrado.

\section{Supplementary Material}

The following online material is available for this article:

Appendix 1 - Excluded species from PFSP species list and the respective reason of exclusion.

Appendix 2 - Number of exclusive species among studies compared with this study.

Appendix 3 - Species occurring at $11(50 \%)$ or more lists among the compared studies and PFSP.

\section{Acknowledgments}

We would like to thank Reinaldo Monteiro and André Luis Teixeira de Lucca for contributing with the flora surveys efforts in PFSP; Jomar Jardim (UFSB), Otávio Luis Marques da Silva (IBt-SP) and Júlio Antonio Lombardi (UNESP) for helping us with some identifications. We are grateful to PFSP staff for supporting fieldwork. 


\section{Author Contributions}

Gabriel Pavan Sabino: elaboration of study's concept and design, data collection, analysis and preparation of manuscritpt;

Gabriel Mendes Marcusso: elaboration of study's concept and design, data collection, analysis and preparation of manuscritpt;

Vitor de Andrade Kamimura: elaboration of study's concept and design, data collection, analysis and preparation of manuscritpt;

Renan Borgiani: elaboration of study's concept, data collection and analysis;

Rafael Konopczyk: elaboration of study's concept and data collection;

Ernesto Pedro Dickfeldt: Contribution to data collection;

José Eduardo de Arruda Bertoni: Contribution to data collection;

Sonia Aparecida de Souza Evangelista: Contribution to data collection.

\section{Conflicts of Interest}

The authors declare that they have no conflict of interest related to the publication of this manuscript.

\section{References}

AB'SÁBER, A.N. 1970. Províncias geológicas e domínios morfoclimáticos no Brasil. IGEOG/USP, São Paulo.

AB'SÁBER, A.N. 2003. Os domínios de natureza do Brasil: Potencialidades paisagísticas. Ateliê Editorial, São Paulo.

ALLEN, T.F.H. \& STARR, T.B. 1982. Hierarchy. Perspectives for ecological complexity. University of Chicago Press, Chicago.

ALVARES, C.A., STAPE, J.L., SENTELHAS, P.C., GONÇALVES, J.L.M. \& SPAROVEK, G. 2013. Köppen's climate classification map for Brazil. Meteorol. Z. 22(6):711-728.

APG IV. 2016. An update of the Angiosperm Phylogeny Group classification for the orders and families of flowering plants: APG IV. Bot. J. Linn. Soc. 181(1):1-20.

BAITELLO, J.B., AGUIAR, O.T., PASTORE, J.A. \& ARZOLLA, F.A.R.D.P.A. 2013. Parque Estadual do Juquery: refúgio de Cerrado no Domínio Atlântico. IF. Ser. Regist. 50:1-46

BARTHLOTT, W., MUTKE, J., RAFIQPOOR, M.D., KIER, G. \& KREFT, H. 2005. Global centers of vascular plant diversity. Nova. Act. Lc. 92:61-83.

BATALHA, M.A. 2011. O cerrado não é um bioma. Biota Neotropica. 11(1):2124. https://www.biotaneotropica.org.br/v11n1/en/abstract?inventory+ bn00111012011 (last access in 01/03/2020)

BATALHA, M.A., ARAGAKI, S. \& MANTOVANI, W. 1997. Florística do cerrado em Emas (Pirassununga, SP). Bol. Bot. Univ. 16:49-64.

BATALHA, M.A. \& MANTOVANI, W. 2001. Floristic composition of the cerrado in the Pé-de-gigante reserve (Santa Rita do Passa Quatro, Southeastern Brazil). Acta Bot. Bras. 15(3):289-304.

BERTONI, J.E.A. 1984. Composição florística e estrutura fitossociológica de uma floresta de interior do Estado de São Paulo: Reserva Estadual de Porto Ferreira. Dissertação de Mestrado, Universidade Estadual de Campinas, Campinas.

BERTONI, J.E.A. \& MARTINS, F.R. 1987. Composição florística de uma floresta ripária na Reserva Estadual de Porto Ferreira, SP. Acta. Bot. Bras. 1(1):17-26

BERTONI, J.E.A., TOLEDO FILHO, D.V., LEITÃO-FILHO, H.F., FRANCO, G.A.D.C. \& AGUIAR, O.T. 2001. Flora arbórea e arbustiva do cerrado do Parque Estadual de Porto Ferreira (SP). Rev. Inst. Florest. 13(2):169-188.

BFG. 2015. Growing knowledge: an overview of Seed Plant diversity in Brazil. Rodriguésia. 66(4):1085-1113.

BFG. 2021. Flora do Brasil 2020. Jardim Botânico do Rio de Janeiro, Rio de Janeiro, p.1-28.
CARVALHO, M.B., ISHARA, K.L. \& RODELLA, R.C.S.M. 2010. Vascular Flora of a Cerrado sensu stricto remnant in Pratânia, state of São Paulo, southeastern Brazil. Check List. 6(3):350-357.

CAVASSAN, O. \& WEISER, V.L. 2015. Vascular flora of the cerrado of BauruSP. Biota Neotropica. 15(3): e20140093. http://dx.doi.org/10.1590/16760611-BN-2014-0093 (last access in 22/11/2020)

CIELO-FILHO, R., BAITELLO, J.B., ARZOLLA, F.A.R.D.P., VILELA, F.E.S.P., KANASHIRO, M.M., MATTOS, I.F.A., AGUIAR, O.T., SOUZA, S.C.P.M., PASTORE, J.A., FRANCO, G.A.D.C. \& ZIMBACK, L. 2015. A vegetação da Estação Ecológica de Avaré: subsídios para o plano de manejo. IF. Ser. Regist. 53:5-42.

COLLI, A.M.T., SOUZA, S.A. \& SILVA, R.T. 2003. Pteridófitas do Parque Estadual de Porto Ferreira (SP), Brasil. Rev. Inst. Flor. 15(11):29-35.

COLLI-SILVA, M., BEZERRA, T.L., FRANCO, G.A.D.C., IVANAUSKAS, N.M. \& SOUZA, F.M. 2016. Registros de espécies vasculares em unidades de conservação e implicações para a lista da flora ameaçada de extinção no estado de São Paulo. Rodriguésia. 67(2):405-425.

COLLI-SILVA, M., IVANAUSKAS, N.M. \& SOUZA, F.M. 2019. Diagnóstico do conhecimento da biodiversidade de plantas vasculares nas unidades de conservação do estado de São Paulo. Rodriguésia. 70:e04582017.

CORRÊA, L.S., SCATIGNA, A.V., GISSI, D.S., SILVA, D.M., COTA, M.M.T., SOUZA, V.C., IVANAUSKAS, N.M., TAMASHIRO, J.Y. \& RODRIGUES, R.R. 2018. Vascular flora checklist of the Ibicatu Ecological Station, Piracicaba, São Paulo, Brazil. Rev. Inst. Flor. 30(1):53-70.

COUTINHO, L.M. 2006. O conceito de bioma. Acta Bot. Bras. 20(1):13-23.

CRIA. 2019. SpeciesLink. http://www.splink.org.br/index (last access in 20/10/2019)

DICKFELDT, E.P., JANDUCCI, B.Z. \& SOUZA, A.S. 2013. Levantamento das espécies vegetais exóticas e experiências de manejo no Parque Estadual de Porto Ferreira, SP. Fórum Ambiental da Alta Paulista. 9(3):118-137.

DRYFLOR, B.R.K., DELGADO-SALINAS, A., DEXTER, K.G., LINARESPALOMINO, R., OLIVEIRA-FILHO, A., PRADO, D. et al. 2016. Plant diversity patterns in neotropical dry forests and their conservation implications. Science. 353(6306):1383-1387.

DURIGAN, G., BACIC, M.C., FRANCO, G.A.D.C. \& SIQUEIRA, M.F. 1999. Inventário florístico do cerrado na Estação Ecológica de Assis, SP. Hoehnea. 26(2):149-172.

DURIGAN, G., RAMOS, V.S., IVANAUSKAS, N.M. \& FRANCO, G.A.D.C. 2012. Espécies indicadoras de fitofisionomias na transição cerrado-mata atlântica no estado de São Paulo. SMA/CBRN, São Paulo.

EISENLOHR, P.V. \& OLIVEIRA-FILHO, A.T. 2015. Revisiting Patterns of Tree Species Composition and their Driving Forces in the Atlantic Forests of Southeastern Brazil. Biotropica. 47(6):689-701.

FERREIRA JÚNIOR, E.V., SOARES, T.S., COSTA, M.F.F., SILVA, V.S.M. 2008. Composição, diversidade e similaridade florística de uma floresta tropical semidecídua submontana em Marcelândia - MT, Brasil. Acta Amaz. 38(4):673-680.

FIASCHI, P. \& PIRANI, J.R. 2009. Review of plant biogeographic studies in Brazil. J. Syst. Evol. 47(5):477-496.

FILGUEIRAS, T.S., NOGUEIRA, P.E., BROCHADO, A.L. \& GUALALL, G.F. 1994. Caminhamento: um método expedito para levantamentos florísticos qualitativos. Cad. Geociênc. 12:39-347.

FLORA DO BRASIL. 2020. Jardim Botânico do Rio de Janeiro. http:// floradobrasil.jbrj.gov.br/ (last access in 12/05/2020)

FORZZA, R.C., PIFANO, D.S., OLIVEIRA-FILHO, A.T., MEIRELES, L.D., FARIA, P.L., SALIMENA, F.R., MYNSSEN, C.M. \& PRADO, J. 2014. Flora vascular da Reserva Biológica da Represa do Grama, Minas Gerais, e sua relação florística com outras florestas do sudeste brasileiro. Rodriguésia. 65(2):275-292.

FRANÇOSO, R.D., HAIDAR, R.F. \& MACHADO, R.B. 2016. Tree species of South America central savanna: endemism, marginal areas and the relationship with other biomes. Acta Bot. Bras. 30(1):78-86.

GOTTSBERGER, G. \& SILBERBAUER-GOTTSBERGER, I. 2006. Life in the Cerrado: a South American Tropical Seasonal Vegetation, Vol. II. Pollination and seed dispersal. Reta Verlag, Ulm. 
GUARATINI, M.T.G., GOMES, E.P.C., TAMASHIRO, J.Y. \& RODRIGUES, R.R. 2008. Composição florística da Reserva Municipal de Santa Genebra, Campinas, SP. Rev. Brasil. Bot. 31(2):323-337.

HARIDASAN, M. 1992. Observations on soils, foliar nutrient concentration and floristic composition of cerrado sensu stricto and cerradão communities in central Brazil. In Nature and Dynamics of Forest: savanna boundaries (P.A. Furley, J. Proctor \& J.A. Ratter, eds). Chapman \& Hall, London, p.171-184.

IBGE. 2004. Mapa de Biomas do Brasil. Diretoria de Geociências, Rio de Janeiro.

IBGE. 2012. Manual técnico da vegetação brasileira. Segunda edição revista e ampliada. Série Manuais Técnicos em Geociências, Rio de Janeiro.

INMET. 2019. http://www.inmet.gov.br/portal/index.php?r=home2/index (last access in 12/10/2019)

INSTITUTO FLORESTAL/FUNDAÇÃO FLORESTAL. 2008. Unidades de Conservação. Arquivos Digitais - APAS Est. SP (CPLA); PESM (ITESP); Mosaico de Jacupiranga; Mosaico da Juréia; Zonas de Amortecimento conforme Planos de Manejos e Áreas Naturais Protegidas do Estado de São Paulo. Florestar Estatístico, v. 11, n. 20.

ISHARA, K.L., DÉSTRO, G.F.G., MAIMONI-RODELLA, R.C.S. \& YANAGIZAWA, Y.A.N.P. 2015. Composição florística de remanescente de cerrado sensu stricto em Botucatu, SP. Rev. Brasil. Bot. 31(4):575-586.

KARK, S. 2012. Ecotones and Ecological Gradients. In Encyclopedia of Sustainability Science and Technology (R. A. Meyers, ed). Springer Science $\&$ Business Media, New York, p.147-160.

KARK S. \& VAN RENSBURG B. J. 2006. Ecotones: marginal or central areas of transition? Isr. J. Ecol. Evol. 52:29-53.

KLINK, C.A. \& MACHADO, R.B. 2005. A conservação do Cerrado Brasileiro. Megadiversidade. 1(1):147-155.

KLINK, C.A. \& MOREIRA, A.G. 2002. Past and current human occupation, and land use. In: The Cerrados of Brazil, OLIVEIRA, P.S. \& MARQUIS, R.J. (eds). Columbia University Press. New York. USA. p 69.

KONOPCZYK, R.M.G. 2014. Composição florística, estrutura e heterogeneidade ambiental da comunidade arbórea de uma várzea em Porto Ferreira, SP, Brasil. Dissertação de Mestrado, Universidade Estadual Paulista, Rio Claro.

LEGENDRE, P. \& LEGENDRE, L. 2012. Numerical Ecology (Vol. 24). 3 ed. Elsevier, Amsterdam.

LIEBERMAN, B.S. 2003. Unifying Theory and Methodology in Biogeography. In Evolutionary Biology (R.J. MacIntyre \& M.T. Clegg, eds). Kluwer Academic/Plenum Publishers, New York, p.1-25.

LOMBARDI, J.A. \& GONÇALVES, M. 2000. Composição florística de dois remanescentes de Mata Atlântica do sudeste de Minas Gerais, Brasil. Rev. Brasil. Bot. 23(3):255-282.

LOMBARDI, J.A., CARVALHO, C.S., BIRAL, L., SAKA, M.N. \& HIEDA, S.M. 2012. Vascular flora of Serra do Japi Biological Reserve, Jundiaí, southeastern Brazil. Rodriguésia. 63(2):333-340.

MARCUSSO, G.M., DICKFELDT, E.P., BERTONI, J.E.A. \& MONTEIRO, R. 2016. Epífitas vasculares do Parque Estadual de Porto Ferreira, São Paulo, Brasil. Rev. Inst. Flor. 28(2):119-133.

MARTINELLI, G. \& MORAES, M.A. (eds). 2013. Livro Vermelho da Flora do Brasil. CNCFlora, Rio de Janeiro.

MENDONCA, R.C., FELFILI, J.M., WALTER, B.M.T., SILVA JÚNIOR, M.C., REZENDE, A.V., FILGUEIRAS, T.S. \& NOGUEIRA, P.E. 2008. Flora vascular do bioma Cerrado: Checklist com 12.356 espécies. In Cerrado: ecologia e flora - vol. 2 (S.M. Samo, S.P. Almeida \& J.F. Ribeiro, eds). Embrapa, Planaltina, p.421-1279.

MITTERMEIER, R.A., ROBLES-GIL, P., HOFFMANN, M., PILGRIM, J., BROOKS, T., MITTERMEIER, C.G., LAMOREUX, J. \& FONSECA, G.A.B. 2004. Hotspots revisited: Earth's biologically richest and most endangered terrestrial ecoregions. EMEX Agrupacíon Sierra Madre, Cidade do México.

MORELLATO, L.P.C. \& HADDAD, C.F. 2000. Introduction: The Brazilian Atlantic Forest. Biotropica. 32(4b):786-792.

MORELLATTO, L.P.C. \& LEITÃO-FILHO, H.F. 1992. Padrões de frutificação e dispersão na Serra do Japi. In História natural da Serra do Japi: ecologia e preservação de uma área florestal no Sudeste do Brasil (L.P.C. Morellatto, ed). Editora da UNICAMP/FAPESP, Campinas, p.112-140.
MORRONE, J.J. 2014. Biogeographical regionalisation of the Neotropical region. Zootaxa. 3782:1-110.

MORRONE, J.J. 2017. Neotropical biogeography: regionalization and evolution. CRC Press, Boca Raton.

MUELLER-DOMBOIS, D. \& ELLENBERG, H. 1974. Aims and methods of vegetation ecology. John Wiley \& Sons, New York.

MYERS, N., MITTERMEIER, R.A., MITTERMEIER, C.G., FONSECA, G.A.B. \& KENT, J. 2000. Biodiversity hotspots for conservation priorities. Nature. 403:852-858.

OKSANEN, J., BLANCHET, F.G., KINDT, R., LEGENDRE, P., MINCHIN, P.R., O'HARA, R.B., SIMPSON, G.L., SOLYMOS, P., STEVENS, M.H.H. \& WAGNER, H. 2013. Vegan: Community Ecology Package. R Package Version 2.0-10. http://vegan.r-forge.r-project.org (last access in $28 / 10 / 2020$ )

OLIVEIRA, A.P.C. 2012. Flora vascular não-arbórea do Parque Estadual de Porto Ferreira, SP, Brasil. Dissertação de Mestrado, Universidade Estadual Paulista, Rio Claro.

OLIVEIRA-FILHO, A.T., BUDKE, J.C., JARENKOW, J.A., EISENLOHR, P.V. \& NEVES, D.R.M. 2015. Delving into the variations in tree species composition and richness across South American subtropical Atlantic and Pampean forests. J. Plant. Ecol. 8(3):242-226.

OLIVEIRA-FILHO, A.T. \& FONTES, M.A.L. 2000. Patterns of Floristic Differentiation among Atlantic Rain Forests in Southeastern Brazil and the Influence of Climate. Biotropica. 32(4b):793-810.

OSACO, M. 2012. Florística e fitossociologia do estrato arbustivo de área detransição savana-floresta no Parque Estadual de Porto Ferreira, SP. Dissertação de Mestrado, Universidade Estadual Paulista, Rio Claro.

PASSOS, F.B., MARIMON, B.S., PHILLIPS, O.L., MORANDI, P.S., NEVES, E.C., ELIAS, F., REIS, S.M., OLIVEIRA, B., FELDPAUSCH, T.R. \& MARIMON JÚNIOR, B.H. 2018. Savanna turning into forest: concerted vegetation change at the ecotone between the Amazon and "Cerrado" biomes. Braz. J. Bot. 41(3):611-619.

PIFANO, D.S., GARCIA, P.O., VALENTE, A.S.M., ANTUNES, K. \& CASTRO, R.M. 2013. Flora fanerogâmica da mata do sítio Primavera na APA Serra dos Núcleos, São João Nepomuceno, Minas Gerais e suas relações florísticas com florestas ombrófilas e semidecíduas no domínio atlântico. Biota. 6(3):12-39.

PINHEIRO, M.H.O., ARANTES, S.A.C.M., JIMENEZ-RUED, J.R. \& MONTEIRO, R. 2009. Caracterização edáfica de um ecótono savânicoflorestal no sudeste brasileiro. Iheringia Ser. Bot. 64(2):15-24.

PINHEIRO, M.H.O. \& MONTEIRO, R. 2008. Florística de uma Floresta Estacional Semidecidual, localizada em ecótono savânico-florestal, no município de Bauru, SP, Brasil. Acta Bot. Bras. 22(4):1085-1094.

PPG I. 2016. A community-derived classification for extant lycophytes and ferns. J. Syst. Evol. 54(6):563-603.

R CORE TEAM. 2020. R: A language and environment for statistical computing. R Foundation for Statistical Computing, Vienna. https://www.R-project.org/ (last access in 02/03/2020)

RATTER, J.A., BRIDGEWATER, S. \& RIBEIRO, J.F. 2003. Analysis of the floristic composition of the Brazilian Cerrado vegetation. III: Comparision of the woody vegetation of 376 areas. Edinb. J. Bot. 60(1):57-109.

RATTER, J.A.; BRIDGEWATER, S.; RIBEIRO, J.F. 2006. Biodiversity patterns of the woody vegetation of the Brazilian Cerrado. In: PENNINGTON, T.; LEWIS GWILYM, P.; RATTER, J.A. (Org.). Neotropical Savannas and dry forests: diversity, biogeography and conservation. Boca Raton: Taylor \& Francis. p.31-66.

RIBEIRO, M.C., METZGER, J.P., MARTENSEN, A.C., PONZONI, F.J. \& HIROTA, M.M. 2009. The Brazilian Atlantic forest: How much is left, and how is the remaining forest distributed? Implications for conservation. Biol. Cons. 142(6):1141-1153.

ROSSETTO, E.F.S. \& VIEIRA, A.O.S. 2013. Vascular Flora of the Mata dos Godoy State Park, Londrina, Paraná, Brazil. Check List. 9(5):1020-1034.

ROSSI, M., MATTOS, I.F.A., COELHO, R.M., MENK, J.R.F., ROCHA, F.T., PFEIFER, R.M. \& DEMARIA, I.C. 2005. Relação solos/vegetação em área natural no Parque Estadual de Porto Ferreira, São Paulo. Rev. Inst. Flor. 17(1):45-61. 
SABINO, G.P. 2013. Florística e Fitossociologia de uma comunidade arbórea em contato savana-floresta no Parque Estadual de Porto Ferreira, Porto Ferreira, São Paulo. Trabalho de Conclusão de Curso, Universidade Estadual Paulista, Rio Claro.

SÃO PAULO. 2003. Plano de manejo do Parque Estadual de Porto Ferreira, Instituto Florestal, São Paulo. https://www.infraestruturameioambiente. sp.gov.br/fundacaoflorestal/planos-de-manejo/planos-de-manejo-planosconcluidos/plano-de-manejo-pe-porto-ferreira/ (last access in 02/03/2020)

SIMON, M.F., GRETHER, R., QUEIROZ, L.P., SKEMA, C., PENNINGTON, R.T. \& HUGHES, C.E. 2009. Recent assembly of the Cerrado, a neotropical plant diversity hotspot, by in situ evolution of adaptations to fire. PNAS. 106(48):20359-20364.

SMA-SP. 2016. Publica a segunda revisão da lista oficial das espécies da flora ameaçadas de extinção no estado de São Paulo. Resolução n ${ }^{\circ} 57$, de 5 de junho de 2016. Secretaria Estadual do Meio Ambiente, São Paulo.

SOS MATA ATLÂNTICA. 2016. Atlas dos remanescentes florestais da Mata Atlântica no período 2014-2015. Fundação SOS Mata Atlântica, São Paulo. http://mapas.sosma.org.br/ (last access in 15/08/2019)

SOUZA, C.R., MOREL, J.D., SANTOS, A.B.M., DA SILVA, W.B., MAIA, V.A., COELHO, P.A., REZENDE, V.L. \& DOS SANTOS, R.M. 2019b. Small-scale edaphic heterogeneity as a floristic-structural complexity driver in Seasonally Dry Tropical Forests tree communities. J. For. Res. 31:2347-2357.

SOUZA, V.C., TOLEDO, C.P., SAMPAIO, D., BÍGIO, N.C., COLLETA, G.D., IVANAUSKAS, N.M. \& FLORES, T.B. 2019a. Guia das plantas da mata atlântica: floresta estacional. Liana Produções Editoriais, Piracicaba.

SOUZA, V.C., FLORES, T.B., COLLETA, G.D. \& COELHO, R.L.G. 2018 Guia das plantas do Cerrado. Editora Taxon Brasil, Piracicaba.

SPICHIGER, R., BISE, B., CALENGE, C. \& CHATELAIN, C. 2006. Biogeography of the forest of the Paraguay-Parana Basin. In Neotropical savannas and seasonally dry forests plant diversity, biogeography, and conservation (R.T. Pennington \& J.A. Ratter, eds). Taylor \& Francis, Boca Raton, p.193-209.
TAVARES, P.A., BRITES, A.D., SPAROVEK, G., GUIDOTTI, V., CERIGNONI, F., AGUIAR, D., METZGER, J.P.W., RODRIGUES, R.R., PINTO, L.F.G., MELLO, K. \& MOLIN, P. G. 2019. Unfolding additional massive cutback effects of the Native Vegetation Protection Law on Legal Reserves, Brazil. Biota Neotropica. 19(4):e20180658. https://doi.org/10.1590/1676-0611bn-2018-0658 (last access in 22/07/2020)

TROPICOS. 2019. http://www.tropicos.org (last access in 25/10/2019)

VARGAS, B.C., OLIVEIRA, A.P.C., UDULUTSCH, R.G., MARCUSSO, G. M., SABINO, G.P., MELO, P.H.A., GRILlO, R.M.M., KAMIMURA, V.A. \& ASSIS, M.A. 2018. Climbing plants of Porto Ferreira State Park, southeastern Brazil. Biota Neotropica. 18(2):e20170346. https://doi. org/10.1590/1676-0611-bn-2017-0346 (last access in 10/02/2020)

VELAZCO, S.J.E., GALVÃO, F., KELLER, H.A. \& BEDRIJ, N.A. 2018. Cerrados in Argentina? Structure, diversity and biogeography of the woody component of a savanna in the Misiones Province. Rodriguésia. 69(2):335-349

VELOSO, H.P. \& GÓES FILHO, L. 1982. Fitogeografia brasileira-classificação fisionômicoecológica da vegetação neotropical. IBGE/Projeto Radambrasil, Salvador.

VIEIRA, L.T.A., POLISEL R.T., IVANAUSKAS, N.M., SHEPHERD, G.J., WAECHTER, J.L., YAMAMOTO, K. \& MARTINS, F.R. 2015. Geographical patterns of terrestrial herbs: a new component in planning the conservation of the Brazilian Atlantic Forest. Biodivers. Conserv. 24(9):2181-2198.

ZANOTTI, C.A. KELLER, H.A. \& ZULOAGA, F.O. 2020. Biodiversidad de la flora vascular de la provincia de Misiones, Región Paranaense Argentina. Darwiniana. 8(1):42-291. 